\title{
On the Use of Lie Group Homomorphisms for Treating Similarity Transformations in Nonadiabatic Photochemistry
}

\author{
Benjamin Lasorne \\ CTMM, Institut Charles Gerhardt Montpellier, CNRS/Université Montpellier 2, CC 15001, Place Eugène Bataillon, \\ 34095 Montpellier, France \\ Correspondence should be addressed to Benjamin Lasorne; benjamin.lasorne@univ-montp2.fr
}

Received 25 March 2014; Accepted 22 May 2014; Published 15 July 2014

Academic Editor: Fabien Gatti

Copyright (C) 2014 Benjamin Lasorne. This is an open access article distributed under the Creative Commons Attribution License, which permits unrestricted use, distribution, and reproduction in any medium, provided the original work is properly cited.

A formulation based on Lie group homomorphisms is presented for simplifying the treatment of unitary similarity transformations of Hamiltonian matrices in nonadiabatic photochemistry. A general derivation is provided whereby it is shown that a similarity transformation acting on a traceless, Hermitian matrix through a unitary matrix of $\mathrm{SU}(n)$ is equivalent to the product of a single matrix of $\mathrm{O}\left(n^{2}-1\right)$ by a real vector. We recall how Pauli matrices are the adequate tool when $n=2$ and show how the same is achieved for $n=3$ with Gell-Mann matrices.

\section{Introduction}

The construction of quasidiabatic states capable of reproducing the properties of a limited set of strongly interacting adiabatic states (block or group Born-Oppenheimer approximation) is a central problem in nonadiabatic photochemistry. As discussed in $[1,2]$, this is closely related to the concept of an effective Hamiltonian matrix (quasidiabatic) obtained as the similarity transform of a real diagonal matrix (adiabatic). The reciprocal problem corresponds to the diagonalisation (or block-diagonalisation) of a Hermitian matrix through an invertible transformation. Few-state cases can be parameterised explicitly in terms of rotation angles whereby the operational formulae are made more tractable upon reformulating the transformation within a vector space spanned by a basis set of matrices through a Lie group homomorphism, following the original suggestion of Mead [3], further explored by Yarkony and coworkers [4-7]. The objective of the present work is to provide the noninitiate theoretical chemists with some basic aspects of the required mathematical background underlying this formulation and to lay the foundations of a general treatment of threestate problems where a few helpful tricks are highlighted to make the operational formulae as compact as possible. The approach proposed by Yarkony and coworkers is generalised in terms of Gell-Mann matrices. Their results based on Euler angles are confirmed and an alternative parameterisation based on Cardan angles is proposed.

Pauli matrices [8], originally introduced for treating two-level spin systems in quantum mechanics and further extended to isospin symmetry and quantum electrodynamics, are well-established mathematical tools for treating twostate problems in theoretical chemistry, for example, when applied to nonadiabatic photochemistry involving conical intersections between two electronic states (see, e.g., $[9,10]$ ). This formulation ultimately relies on a Lie group homomorphism from $\mathrm{SU}(2)$ to $\mathrm{SO}(3)$, where the former is a double cover of the latter [11]. In this, traceless, Hermitian matrices are isomorphic to vectors and treated as such, and unitary similarity transformations act on them as rotation matrices act on the isomorphic vectors. Although elegant and compact, this formulation does not provide much more insight than directly separating the trace from the traceless part of a $2 \times 2$ Hamiltonian matrix when considering a similarity transformation (e.g., when diagonalising) and noticing that a rotation of the two states through an angle implies a rotation through twice this angle of the halfdifference and coupling entries. However, it can really make a difference to treat problems with three states or more, as it yields relationships that are much more compact and easier to manipulate, as exemplified by the seminal papers of Yarkony and coworkers on conical intersections with more than two 
electronic states [4-7]. Here, we reanalysed their approach for three-state problems upon examining the properties of GellMann matrices [12], first introduced for describing the colour charge of quarks and gluons in quantum chromodynamics. We propose a trick based on a threefold equivalence to simplify the derivation of the relevant matrices of $\mathrm{O}(8)$.

This paper is written from a theoretical-chemistry perspective and is aimed at nonexperts in the formalism of group theory and linear algebra. It is purposely pedestrian and nonexhaustive, as its objective is to provide operational tools to facilitate the treatment of similarity transforms of Hamiltonian matrices in nonadiabatic photochemistry, where a finite set of electronic states must be considered as coupled. For details on the underlying mathematical foundations, the reader is referred to textbooks on Lie groups and algebras such as, for example, [13].

We first recall some general properties of traceless, Hermitian matrices in the context of Lie group homomorphisms for treating similarity transformations and illustrate this with Pauli matrices. Then, we focus on Gell-Mann matrices and provide some practical examples showing how this formulation can prove useful when dealing with three-state Hamiltonian matrices.

\section{Lie Group Homomorphisms for Similarity Transformations}

Let $n \in \mathbb{N}^{*}$. Any $n \times n$ complex matrix $\mathbf{M} \in M_{n}(\mathbb{C})$ can be uniquely expanded as

$$
\mathbf{M}=\frac{\operatorname{tr}(\mathbf{M})}{n} \mathbf{1}+\overline{\mathbf{M}}
$$

where $\mathbf{1}$ is the identity matrix of rank $n$ and $\overline{\mathbf{M}}$ is the traceless part of $\mathbf{M}$. As $M_{n}(\mathbb{C})$ is a complex vector space (a vector space over the scalar field of complex numbers, $\mathbb{C}$ ) with respect to matrix addition and scalar multiplication, it is possible to define a complete and linearly independent set of $n^{2}$ basis matrices, $\mathscr{B}=\left\{\mathbf{1}, \boldsymbol{\beta}_{m} ; m=1, \ldots, n^{2}-1\right\}$, such that

$$
\mathbf{M}=\mathscr{M}_{0} \mathbf{1}+\sum_{m=1}^{n^{2}-1} \mathscr{M}_{m} \boldsymbol{\beta}_{m},
$$

where the $n^{2}$ entries of $\mathscr{M}=\left(\mathscr{M}_{0}, \mathscr{M}_{m} ; m=1, \ldots, n^{2}-1\right)^{\mathrm{T}} \epsilon$ $\mathbb{C}^{n^{2}}$ and the $n^{2}$ entries of $\mathbf{M} \in M_{n}(\mathbb{C})$ are related through an isomorphism that depends on the particular choice made for the basis set. In what follows, bold scripts such as $\mathscr{M}$ will be used to denote the corresponding column-vectors (and $\overline{\mathscr{M}}$ when $\mathscr{M}_{0}$ is excluded). Note that, hereafter, we will deliberately identify the $n^{2}$-tuple vectors of $\mathbb{C}^{n^{2}}$ to the isomorphic $n^{2}$-line column-vectors of $M_{n^{2}, 1}(\mathbb{C})$ for notation simplicity.

The complex Frobenius (also known as Hilbert-Schmidt) inner product,

$$
\begin{gathered}
\forall \mathbf{M}_{a}, \mathbf{M}_{b} \in M_{n}(\mathbb{C}), \\
\mathbf{M}_{a}: \mathbf{M}_{b}=\operatorname{tr}\left(\mathbf{M}_{a}^{\dagger} \mathbf{M}_{b}\right)=\operatorname{tr}\left(\mathbf{M}_{b}^{\dagger} \mathbf{M}_{a}\right)^{*}=\left(\mathbf{M}_{b}: \mathbf{M}_{a}\right)^{*}
\end{gathered}
$$

defines a Hermitian metric, with respect to which the basis set $\mathscr{B}$ can be chosen orthogonal,

$$
\begin{gathered}
\mathbf{1}: \mathbf{1}=\operatorname{tr}(\mathbf{1})=n, \\
\mathbf{1}: \boldsymbol{\beta}_{m}=\boldsymbol{\beta}_{m}: \mathbf{1}=0, \\
\boldsymbol{\beta}_{m}: \boldsymbol{\beta}_{m^{\prime}}=\boldsymbol{\beta}_{m^{\prime}}: \boldsymbol{\beta}_{m}=2 \delta_{m m^{\prime}},
\end{gathered}
$$

where $\delta_{m m^{\prime}}$ is the Kronecker symbol. This implies that the $\boldsymbol{\beta}_{m^{-}}$ matrices are traceless, since $1: \boldsymbol{\beta}_{m}=\operatorname{tr}\left(\boldsymbol{\beta}_{m}\right)$. In addition, we choose the Hermitian, that is, $\boldsymbol{\beta}_{m}^{\dagger}=\boldsymbol{\beta}_{m}$. Note that they are not normalised and would be so if multiplied by a factor $1 / \sqrt{2}$ (and the identity by a factor $1 / \sqrt{n}$ ). The weight factor 2 is conventional and chosen according to the definition of Pauli and Gell-Mann matrices (see below). Any other homogeneous scaling factor would work just as well. The corresponding closure relationship reads

$$
\mathbf{M}=\frac{\mathbf{1}: \mathbf{M}}{n} \mathbf{1}+\sum_{m=1}^{n^{2}-1} \frac{\boldsymbol{\beta}_{m}: \mathbf{M}}{2} \boldsymbol{\beta}_{m},
$$

such that the entries of $\mathscr{M}$ satisfy

$$
\begin{gathered}
\mathscr{M}_{0}=\frac{\mathbf{1}: \mathbf{M}}{n}=\frac{\operatorname{tr}(\mathbf{M})}{n}, \\
\mathscr{M}_{m}=\frac{\boldsymbol{\beta}_{m}: \mathbf{M}}{2}=\frac{\operatorname{tr}\left(\boldsymbol{\beta}_{m} \mathbf{M}\right)}{2} .
\end{gathered}
$$

It is also true that $\overline{\mathbf{M}}$ is isomorphic to $\overline{\mathscr{M}}=\left(\mathscr{M}_{m} ; m=1, \ldots\right.$, $\left.n^{2}-1\right)^{\mathrm{T}} \in \mathbb{C}^{n^{2}-1}$.

Let us now consider any $n \times n$ complex Hermitian matrix $\mathbf{H} \in H_{n}(\mathbb{C})$, where

$$
H_{n}(\mathbb{C})=\left\{\mathbf{H} \in M_{n}(\mathbb{C}) \mid \mathbf{H}^{\dagger}=\mathbf{H}\right\} .
$$

The previous properties hold, except that all entries of $\mathscr{H}$ are now real. This defines an isomorphism between $H_{n}(\mathbb{C})$ and $\mathbb{R}^{n^{2}}$ and a similar isomorphism between $\bar{H}_{n}(\mathbb{C})$ and $\mathbb{R}^{n^{2}-1}$, where

$$
\bar{H}_{n}(\mathbb{C})=\left\{\overline{\mathbf{H}} \in H_{n}(\mathbb{C}) \mid \operatorname{tr}(\overline{\mathbf{H}})=0\right\}
$$

$\bar{H}_{n}(\mathbb{C})$, the set of traceless, $n \times n$ complex Hermitian matrices, is thus an $\left(n^{2}-1\right)$-dimensional real vector space (a vector space over the scalar field of real numbers, $\mathbb{R}$ ) with respect to matrix addition and scalar multiplication,

$$
\begin{gathered}
\forall \overline{\mathbf{H}}_{a}, \overline{\mathbf{H}}_{b} \in \bar{H}_{n}(\mathbb{C}), \quad \forall \lambda \in \mathbb{C}, \\
\left(\overline{\mathbf{H}}_{a}+\lambda \overline{\mathbf{H}}_{b}\right)^{\dagger}=\overline{\mathbf{H}}_{a}^{\dagger}+\lambda^{*} \overline{\mathbf{H}}_{b}^{\dagger}=\overline{\mathbf{H}}_{a}+\lambda^{*} \overline{\mathbf{H}}_{b} \\
\Longrightarrow\left\{\overline{\mathbf{H}}_{a}+\lambda \overline{\mathbf{H}}_{b} \in \bar{H}_{n}(\mathbb{C}) \Longleftrightarrow \lambda \in \mathbb{R}\right\}, \\
\operatorname{tr}\left(\overline{\mathbf{H}}_{a}+\lambda \overline{\mathbf{H}}_{b}\right)=\operatorname{tr}\left(\overline{\mathbf{H}}_{a}\right)+\lambda \operatorname{tr}\left(\overline{\mathbf{H}}_{b}\right)=0 .
\end{gathered}
$$


$\overline{\mathscr{B}}=\left\{\boldsymbol{\beta}_{m} ; m=1, \ldots, n^{2}-1\right\}$ is a complete and orthogonal basis set of $\bar{H}_{n}(\mathbb{C})$ and $\bar{H}_{n}(\mathbb{C})$ is a Euclidean space with respect to the, now real, Frobenius inner product,

$$
\begin{gathered}
\forall \overline{\mathbf{H}}_{a}, \overline{\mathbf{H}}_{b} \in \bar{H}_{n}(\mathbb{C}), \\
\overline{\mathbf{H}}_{a}: \overline{\mathbf{H}}_{b}=\operatorname{tr}\left(\overline{\mathbf{H}}_{a} \overline{\mathbf{H}}_{b}\right)=\operatorname{tr}\left(\overline{\mathbf{H}}_{b} \overline{\mathbf{H}}_{a}\right)=\overline{\mathbf{H}}_{b}: \overline{\mathbf{H}}_{a},
\end{gathered}
$$

which, upon expanding over $\overline{\mathscr{B}}$ and halving, canonically identifies the standard Euclidean dot product of $\mathbb{R}^{n^{2}-1}$,

$$
\frac{\overline{\mathbf{H}}_{a}: \overline{\mathbf{H}}_{b}}{2}=\sum_{m=1}^{n^{2}-1} \mathscr{H}_{a m} \mathscr{H}_{b m}=\overline{\mathscr{H}}_{a} \cdot \overline{\mathscr{H}}_{b}=\overline{\mathscr{H}}_{a}^{\mathrm{T}} \overline{\mathscr{H}}_{b} .
$$

In addition, $\bar{H}_{n}(\mathbb{C})$ is related through matrix exponentiation to the $n$-fold special unitary Lie group $\mathrm{SU}_{n}(\mathbb{C}) \equiv \mathrm{SU}(n)$, for which the basis set of traceless, skew-Hermitian matrices, $\left\{i \boldsymbol{\beta}_{m} ; m=1, \ldots, n^{2}-1\right\}$, is a possible representation of the infinitesimal generators belonging to the corresponding Lie algebra, $\operatorname{su}(n)$. For $n=2$ and 3 we will consider $\boldsymbol{\beta}_{m}$ as the three Pauli matrices, $\sigma_{m}$, and the eight Gell-Mann matrices, $\lambda_{m}$, respectively, (see next sections).

Now, let us turn to unitary similarity transformations of Hermitian matrices. Through any unitary matrix $\mathbf{U} \in$ $\mathrm{U}_{n}(\mathbb{C}) \equiv \mathrm{U}(n)$, that is, $\mathbf{U}^{-1}=\mathbf{U}^{\dagger}$, and for any $\mathbf{H} \in H_{n}(\mathbb{C})$, the similarity transform, $\mathbf{U}^{-1} \mathbf{H U}=\mathbf{U}^{\dagger} \mathbf{H U}$, is Hermitian and breaks into

$$
\mathbf{U}^{\dagger} \mathbf{H U}=\mathscr{H}_{0} \mathbf{1}+\mathbf{U}^{\dagger} \overline{\mathbf{H}} \mathbf{U}
$$

where $\mathscr{H}_{0}=\operatorname{tr}\left(\mathbf{U}^{\dagger} \mathbf{H U}\right) / n=\operatorname{tr}(\mathbf{H}) / n$; that is, $\operatorname{tr}\left(\mathbf{U}^{\dagger} \overline{\mathbf{H}} \mathbf{U}\right)=0$. The trace is preserved, and the only practical difficulty from an operational perspective lies in transforming the traceless part. As both $\overline{\mathbf{H}}$ and $\mathbf{U}^{\dagger} \overline{\mathbf{H}} \mathbf{U}$ are Hermitian and traceless too, the similarity transformation defines a linear map of the real vector space $\bar{H}_{n}(\mathbb{C})$,

$$
\begin{gathered}
\forall \overline{\mathbf{H}}_{a}, \overline{\mathbf{H}}_{b} \in \bar{H}_{n}(\mathbb{C}), \quad \forall \lambda \in \mathbb{R}, \\
\mathbf{U}^{\dagger}\left(\overline{\mathbf{H}}_{a}+\lambda \overline{\mathbf{H}}_{b}\right) \mathbf{U}=\mathbf{U}^{\dagger}\left(\overline{\mathbf{H}}_{a}\right) \mathbf{U}+\lambda \mathbf{U}^{\dagger}\left(\overline{\mathbf{H}}_{b}\right) \mathbf{U} \in \bar{H}_{n}(\mathbb{C}),
\end{gathered}
$$

such that, for $\overline{\mathbf{H}}^{\prime}=\mathbf{U}^{\dagger} \overline{\mathbf{H}} \mathbf{U}$ and its isomorphic column-vector $\overline{\mathscr{H}}^{\prime}$, there exists a unique, real $\left(n^{2}-1\right) \times\left(n^{2}-1\right)$ matrix $\mathscr{R} \epsilon$ $M_{n^{2}-1}(\mathbb{R})$ that satisfies

$$
\overline{\mathbf{H}}^{\prime}=\sum_{m=1}^{n^{2}-1} \mathscr{H}_{m}^{\prime} \boldsymbol{\beta}_{m}=\sum_{m=1}^{n^{2}-1} \sum_{m^{\prime}=1}^{n^{2}-1}\left(\mathscr{R}_{m m^{\prime}} \mathscr{H}_{m^{\prime}}\right) \boldsymbol{\beta}_{m} ;
$$

that is,

$$
\left\{\begin{array} { l } 
{ \overline { H } _ { n } ( \mathbb { C } ) \longrightarrow \overline { H } _ { n } ( \mathbb { C } ) } \\
{ \overline { \mathbf { H } } \longmapsto \overline { \mathbf { H } } ^ { \prime } = \mathbf { U } ^ { \dagger } \overline { \mathbf { H } } \mathbf { U } }
\end{array} \longleftrightarrow \left\{\begin{array}{c}
\mathbb{R}^{n^{2}-1} \longrightarrow \mathbb{R}^{n^{2}-1} \\
\overline{\mathscr{H}} \longmapsto \overline{\mathscr{H}}^{\prime}=\mathscr{R} \overline{\mathscr{H}} .
\end{array}\right.\right.
$$

This is the first central idea of the formulation exposed here and in the papers of Yarkony and coworkers [4-7].
The product of three $n \times n$ complex matrices required to evaluate the similarity transform is readily expressed as an invariant trace augmented with the product of an $\left(n^{2}-1\right) \times$ $\left(n^{2}-1\right)$ real matrix by an $\left(n^{2}-1\right)$ real vector. Although this may not be more efficient computationally in all cases, the corresponding expressions are less entangled.

Let us now examine the properties of $\mathscr{R}$. The similarity transformation preserves the trace through matrix product,

$$
\begin{gathered}
\forall \overline{\mathbf{H}}_{a}, \overline{\mathbf{H}}_{b} \in \bar{H}_{n}(\mathbb{C}), \\
\operatorname{tr}\left(\mathbf{U}^{\dagger} \overline{\mathbf{H}}_{a} \mathbf{U} \mathbf{U}^{\dagger} \overline{\mathbf{H}}_{b} \mathbf{U}\right)=\operatorname{tr}\left(\mathbf{U}^{\dagger} \overline{\mathbf{H}}_{a} \overline{\mathbf{H}}_{b} \mathbf{U}\right)=\operatorname{tr}\left(\overline{\mathbf{H}}_{a} \overline{\mathbf{H}}_{b}\right),
\end{gathered}
$$

using the cyclicity of the trace. In other words, it preserves the inner product of $\bar{H}_{n}(\mathbb{C})$,

$$
\mathbf{U}^{\dagger} \overline{\mathbf{H}}_{a} \mathbf{U}: \mathbf{U}^{\dagger} \overline{\mathbf{H}}_{b} \mathbf{U}=\overline{\mathbf{H}}_{a}: \overline{\mathbf{H}}_{b}
$$

and is thus an isometry with respect to the metric of this vector space. From the isomorphism between $\bar{H}_{n}(\mathbb{C})$ and $\mathbb{R}^{n^{2}-1}$, we get

$$
\overline{\mathscr{H}}_{a}^{\prime} \cdot \overline{\mathscr{H}}_{b}^{\prime}=\frac{\mathbf{U}^{\dagger} \overline{\mathbf{H}}_{a} \mathbf{U}: \mathbf{U}^{\dagger} \overline{\mathbf{H}}_{b} \mathbf{U}}{2}=\frac{\overline{\mathbf{H}}_{a}: \overline{\mathbf{H}}_{b}}{2}=\overline{\mathscr{H}}_{a} \cdot \overline{\mathscr{H}}_{b} ;
$$

that is,

$$
\begin{aligned}
\left(\mathscr{R} \overline{\mathscr{H}}_{a}\right) \cdot\left(\mathscr{R} \overline{\mathscr{H}}_{b}\right) & =\overline{\mathscr{H}}_{a} \cdot \overline{\mathscr{H}}_{b}, \\
\overline{\mathscr{H}}_{a}^{\mathrm{T}}\left(\mathscr{R}^{\mathrm{T}} \mathscr{R}\right) \overline{\mathscr{H}}_{b} & =\overline{\mathscr{H}}_{a}^{\mathrm{T}} \overline{\mathscr{H}}_{b},
\end{aligned}
$$

and $\mathscr{R}$ is thus an orthogonal matrix; that is, $\mathscr{R}^{-1}=\mathscr{R}^{\mathrm{T}}$ and $\mathscr{R} \in \mathrm{O}_{n^{2}-1}(\mathbb{R}) \equiv \mathrm{O}\left(n^{2}-1\right)$. The explicit expression of $\mathscr{R}$ can be obtained from the transformation of $\overline{\mathscr{B}}$, as any $\boldsymbol{\beta}_{m}$ is isomorphic to the corresponding canonical basis vector of $\mathbb{R}^{n^{2}-1}, \boldsymbol{e}_{m}=\left(\delta_{m^{\prime} m} ; m^{\prime}=1, \ldots, n^{2}-1\right)^{\mathrm{T}}$. Hence,

$$
\mathbf{U}^{\dagger} \boldsymbol{\beta}_{m} \mathbf{U}=\sum_{m^{\prime}=1}^{n^{2}-1} \boldsymbol{\beta}_{m^{\prime}} \mathscr{R}_{m^{\prime} m}
$$

that is,

$$
\mathscr{R}_{m^{\prime} m}=\frac{\boldsymbol{\beta}_{m^{\prime}}:\left(\mathbf{U}^{\dagger} \boldsymbol{\beta}_{m} \mathbf{U}\right)}{2} .
$$

In other words, each column $m$ of $\mathscr{R}$, obtained as $\mathscr{R} \boldsymbol{e}_{m}$, is made of the components of $\mathbf{U}^{\dagger} \boldsymbol{\beta}_{m} \mathbf{U}$ with respect to $\overline{\mathscr{B}}$. mation,

The map corresponding to the unitary similarity transfor-

$$
\begin{aligned}
\mathrm{U}(n) & \longrightarrow \mathrm{O}\left(n^{2}-1\right), \\
\mathbf{U}^{\dagger} & \longrightarrow \mathscr{R},
\end{aligned}
$$

is a group homomorphism. The image of $\mathbf{1}$ in $\mathrm{U}(n)$ is $\mathbf{1}$ in $\mathrm{O}\left(n^{2}-1\right)$, as

$$
\boldsymbol{\beta}_{m}=\sum_{m^{\prime}=1}^{n^{2}-1} \boldsymbol{\beta}_{m^{\prime}} \delta_{m^{\prime} m}
$$


The image of the product is the product of the images,

$$
\begin{gathered}
\forall \mathbf{U}_{a}, \mathbf{U}_{b} \in \mathbf{U}(n), \\
\mathbf{U}_{a}^{\dagger} \longmapsto \mathscr{R}_{a} \Longrightarrow \mathbf{U}_{a}^{\dagger} \boldsymbol{\beta}_{m} \mathbf{U}_{a}=\sum_{m^{\prime}=1}^{n^{2}-1} \boldsymbol{\beta}_{m^{\prime}} \mathscr{R}_{a m^{\prime} m}, \\
\mathbf{U}_{b}^{\dagger} \longmapsto \mathscr{R}_{b} \Longrightarrow \mathbf{U}_{b}^{\dagger} \boldsymbol{\beta}_{m} \mathbf{U}_{b}=\sum_{m^{\prime}=1}^{n^{2}-1} \boldsymbol{\beta}_{m^{\prime}} \mathscr{R}_{b m^{\prime} m}, \\
\mathbf{U}_{a}^{\dagger} \mathbf{U}_{b}^{\dagger} \longmapsto \mathscr{R}_{a} \mathscr{R}_{b},
\end{gathered}
$$

since

$$
\begin{aligned}
\mathbf{U}_{a}^{\dagger} \mathbf{U}_{b}^{\dagger} \boldsymbol{\beta}_{m} \mathbf{U}_{b} \mathbf{U}_{a} & =\sum_{m^{\prime \prime}=1}^{n^{2}-1} \mathbf{U}_{a}^{\dagger} \boldsymbol{\beta}_{m^{\prime \prime}} \mathbf{U}_{a} \mathscr{R}_{b m^{\prime \prime} m} \\
& =\sum_{m^{\prime}=1}^{n^{2}-1} \boldsymbol{\beta}_{m^{\prime}}\left(\mathscr{R}_{a} \mathscr{R}_{b}\right)_{m^{\prime} m}
\end{aligned}
$$

where

$$
\left(\mathscr{R}_{a} \mathscr{R}_{b}\right)_{m^{\prime} m}=\sum_{m^{\prime \prime}=1}^{n^{2}-1} \mathscr{R}_{a m^{\prime} m^{\prime \prime}} \mathscr{R}_{b m^{\prime \prime} m}
$$

One, thus, deduces that the image of the inverse is the inverse of the image,

$$
\forall \mathbf{U} \in \mathrm{U}(n), \quad \mathbf{U}^{\dagger} \longmapsto \mathscr{R} \Longrightarrow\left(\mathbf{U}^{\dagger}\right)^{-1}=\mathbf{U} \longmapsto \mathscr{R}^{-1}=\mathscr{R}^{\mathrm{T}}
$$

This is the second central idea of this formulation: if $\mathbf{U}^{\dagger}$ is decomposed as a product of elementary unitary matrices, the corresponding orthogonal matrix $\mathscr{R}$ will simply be the product of their images. An operational parameterisation of such a transformation in terms of a set of angles is thus betterfactorised, as each angle appears only once (in a single matrix factor $\mathscr{R}_{a}$ contained in $\mathscr{R}$ ) rather than twice (in a two matrix factors contained both in $\mathbf{U}_{a}^{\dagger}$ and $\mathbf{U}_{a}$ ).

This group homomorphism is not an isomorphism. Indeed, for any $\alpha \in \mathbb{R}$, since $\left[e^{i \alpha} \mathbf{U}\right]^{\dagger}=e^{-i \alpha} \mathbf{U}^{\dagger}$, then $\mathbf{U}^{\dagger} \mapsto \mathscr{R}$ and $e^{-i \alpha} \mathbf{U}^{\dagger} \mapsto \mathscr{R}$. The "useful" set of unitary matrices can obviously be restricted to $\mathrm{SU}(n)$ by getting rid of the complex phases of their determinants, which is of no consequence on the similarity transform. However, there still are $n$ distinct matrices of $\mathrm{SU}(n)$ sharing the same image. Indeed,

$$
\begin{gathered}
\forall \mathbf{U} \in \operatorname{SU}(n), \\
\operatorname{det}(\mathbf{U})=\operatorname{det}\left(\mathbf{U}^{\dagger}\right)=1 \\
\Longrightarrow\left\{\forall k=1, \ldots, n-1: e^{i(2 \pi k / n)} \mathbf{U}\right. \\
\neq \mathbf{U} ; \operatorname{det}\left(e^{i(2 \pi k / n)} \mathbf{U}\right) \\
\left.=\operatorname{det}\left(e^{-i(2 \pi k / n)} \mathbf{U}^{\dagger}\right)=1\right\} .
\end{gathered}
$$

In the following examples with $n=2$ and 3 , we will further consider restrictions of complex matrices of $\mathrm{SU}(n)$ to real matrices of $\mathrm{SO}(n)$ when similarity transformations are limited to rotations applied to real Hermitian matrices.

\section{Pauli Matrices for Two-State Hamiltonian Matrices}

We now recall some properties of the well-known Pauli matrices $\left(n=2, \boldsymbol{\beta}_{m} \equiv \boldsymbol{\sigma}_{m}\right.$ ) that can prove elegant, if not useful, in the context of two-electronic-state problems in nonadiabatic photochemistry. The three Pauli matrices are traceless, Hermitian, and defined as

$$
\boldsymbol{\sigma}_{1}=\left[\begin{array}{ll}
0 & 1 \\
1 & 0
\end{array}\right], \quad \boldsymbol{\sigma}_{2}=\left[\begin{array}{cc}
0 & -i \\
i & 0
\end{array}\right], \quad \boldsymbol{\sigma}_{3}=\left[\begin{array}{cc}
1 & 0 \\
0 & -1
\end{array}\right] .
$$

Thus, the corresponding $\mathbf{M} \in M_{2}(\mathbb{C}) \mapsto \mathscr{M} \in \mathbb{C}^{4}$ isomorphism is such that

$$
\begin{aligned}
\mathbf{M} & =\left[\begin{array}{ll}
M_{11} & M_{12} \\
M_{21} & M_{22}
\end{array}\right] \\
& =\left[\begin{array}{ll}
\mathscr{M}_{0}+\mathscr{M}_{3} & \mathscr{M}_{1}-i \mathscr{M}_{2} \\
\mathscr{M}_{1}+i \mathscr{M}_{2} & \mathscr{M}_{0}-\mathscr{M}_{3}
\end{array}\right] \\
& \longleftrightarrow \mathscr{M} \\
& =\left[\begin{array}{l}
\mathscr{M}_{0} \\
\mathscr{M}_{1} \\
\mathscr{M}_{2} \\
\mathscr{M}_{3}
\end{array}\right]=\left[\begin{array}{l}
\frac{M_{11}+M_{22}}{2} \\
\frac{M_{12}+M_{21}}{2} \\
i \frac{M_{12}-M_{21}}{2} \\
\frac{M_{11}-M_{22}}{2}
\end{array}\right] .
\end{aligned}
$$

For a complex Hermitian matrix $\mathbf{H}$, we get four real parameters, as required: $\mathscr{H}_{0}$ and

$$
\overline{\mathscr{H}}=\left[\begin{array}{c}
\mathscr{H}_{1} \\
\mathscr{H}_{2} \\
\mathscr{H}_{3}
\end{array}\right]=\left[\begin{array}{c}
\mathfrak{R} H_{21}=\mathfrak{R} H_{12} \\
\mathfrak{J} H_{21}=-\mathfrak{J} H_{12} \\
\frac{H_{11}-H_{22}}{2}
\end{array}\right] .
$$

Pauli matrices satisfy, for any $m_{1}, m_{2} \in\{1,2,3\}$,

$$
\boldsymbol{\sigma}_{m_{1}} \boldsymbol{\sigma}_{m_{2}}=\delta_{m_{1} m_{2}} \mathbf{1}+i \sum_{m_{3}=1}^{3} \epsilon_{m_{1} m_{2} m_{3}} \boldsymbol{\sigma}_{m_{3}},
$$

where $\epsilon_{m_{1} m_{2} m_{3}}$ is the threefold Levi-Civita symbol. This is summarised in Table 1.

As a consequence,

$$
\operatorname{tr}\left(\boldsymbol{\sigma}_{m_{1}} \boldsymbol{\sigma}_{m_{2}}\right)=2 \delta_{m_{1} m_{2}}
$$

and, for any $m_{1}, m_{2}, m_{3} \in\{1,2,3\}$,

$$
\operatorname{tr}\left(\boldsymbol{\sigma}_{m_{1}} \boldsymbol{\sigma}_{m_{2}} \boldsymbol{\sigma}_{m_{3}}\right)=2 i \epsilon_{m_{1} m_{2} m_{3}} .
$$


TABLE 1: Multiplication table of Pauli matrices (left times top).

\begin{tabular}{lccc}
\hline Product & $\boldsymbol{\sigma}_{1}$ & $\boldsymbol{\sigma}_{2}$ & $\boldsymbol{\sigma}_{3}$ \\
\hline $\boldsymbol{\sigma}_{1}$ & $\mathbf{1}$ & $i \boldsymbol{\sigma}_{3}$ & $-i \boldsymbol{\sigma}_{2}$ \\
$\boldsymbol{\sigma}_{2}$ & $-i \boldsymbol{\sigma}_{3}$ & $\mathbf{1}$ & $i \boldsymbol{\sigma}_{1}$ \\
$\boldsymbol{\sigma}_{3}$ & $i \boldsymbol{\sigma}_{2}$ & $-i \boldsymbol{\sigma}_{1}$ & $\mathbf{1}$ \\
\hline
\end{tabular}

In the case $n=2$, it is easy to demonstrate that, for any given U such that $\mathbf{U}^{\dagger} \mapsto \mathscr{R}$, the determinant of $\mathscr{R}$ is one and $\mathscr{R}$ is a rotation matrix. First, let us notice that

$$
\operatorname{tr}\left(\boldsymbol{\sigma}_{m_{1}} \boldsymbol{\sigma}_{m_{2}} \boldsymbol{\sigma}_{m_{3}}\right)=\epsilon_{m_{1} m_{2} m_{3}} \operatorname{tr}\left(\boldsymbol{\sigma}_{1} \boldsymbol{\sigma}_{2} \boldsymbol{\sigma}_{3}\right)
$$

Then,

$$
\begin{aligned}
\operatorname{tr}\left(\boldsymbol{\sigma}_{1} \boldsymbol{\sigma}_{2} \boldsymbol{\sigma}_{3}\right) \\
=\operatorname{tr}\left(\mathbf{U}^{\dagger} \boldsymbol{\sigma}_{1} \mathbf{U U}^{\dagger} \boldsymbol{\sigma}_{2} \mathbf{U} \mathbf{U}^{\dagger} \boldsymbol{\sigma}_{3} \mathbf{U}\right) \\
=\operatorname{tr}\left[\left(\sum_{m_{1}=1}^{3} \boldsymbol{\sigma}_{m_{1}} \mathscr{R}_{m_{1} 1}\right)\left(\sum_{m_{2}=1}^{3} \boldsymbol{\sigma}_{m_{2}} \mathscr{R}_{m_{2} 2}\right)\right. \\
\left.\quad \times\left(\sum_{m_{3}=1}^{3} \boldsymbol{\sigma}_{m_{3}} \mathscr{R}_{m_{3} 3}\right)\right] \\
=\sum_{m_{1}=1}^{3} \sum_{m_{2}=1 m_{3}=1}^{3} \sum_{m_{1} 1}^{3} \mathscr{R}_{m_{2} 2} \mathscr{R}_{m_{3} 3} \operatorname{tr}\left(\boldsymbol{\sigma}_{m_{1}} \boldsymbol{\sigma}_{m_{2}} \boldsymbol{\sigma}_{m_{3}}\right) \\
=\operatorname{tr}\left(\boldsymbol{\sigma}_{1} \boldsymbol{\sigma}_{2} \boldsymbol{\sigma}_{3}\right) \sum_{m_{1}=1 m_{2}=1 \sum_{m_{3}=1}^{3} \epsilon_{m_{1} m_{2} m_{3}}^{3} \mathscr{R}_{m_{1} 1} \mathscr{R}_{m_{2} 2} \mathscr{R}_{m_{3} 3}}^{3} \\
=\operatorname{tr}\left(\boldsymbol{\sigma}_{1} \boldsymbol{\sigma}_{2} \boldsymbol{\sigma}_{3}\right) \operatorname{det}(\mathscr{R}) .
\end{aligned}
$$

As $\operatorname{tr}\left(\sigma_{1} \sigma_{2} \sigma_{3}\right)=2 i \neq 0$, then $\operatorname{det}(\mathscr{R})=1$. The aforementioned group homomorphism $\mathrm{U}^{\dagger} \in \mathrm{U}(2) \mapsto \mathscr{R} \in \mathrm{O}(3)$ can thus be restricted to $\mathrm{U}(2) \rightarrow \mathrm{SO}(3)$ and, in practice, to $\mathrm{SU}(2) \rightarrow \mathrm{SO}(3)$. The latter is of kernel $\{ \pm \mathbf{1}\}$ such that $\mathrm{SU}(2)$ is known as a double cover of $\mathrm{SO}(3): \pm \mathbf{U}^{\dagger} \mapsto \mathscr{R}$. This result formally reflects the invariance of half-unit spins through $4 \pi$-rotation and the Berry geometrical phase (also known as molecular Bohm-Aharonov effect) in two-state systems $[3,9,10,14-16]$.

Let us now consider the particular example of a direct rotation through an angle $\varphi$, represented with the following orthogonal matrix:

$$
\mathbf{U}(\varphi)=\left[\begin{array}{cc}
\cos \varphi & -\sin \varphi \\
\sin \varphi & \cos \varphi
\end{array}\right]
$$

It can be expressed from matrix exponentiation of $-i \varphi \sigma_{2}$ as

$$
\mathbf{U}(\varphi)=e^{-i \varphi \boldsymbol{\sigma}_{2}}=\cos \varphi \mathbf{1}-i \sin \varphi \boldsymbol{\sigma}_{2}
$$

and is such that $\mathbf{U}^{-1}(\varphi)=\mathbf{U}^{\dagger}(\varphi)=\mathbf{U}^{\mathrm{T}}(\varphi)=\mathbf{U}(-\varphi)$. The corresponding similarity transformation satisfies for each basis matrix

$$
\begin{gathered}
e^{i \varphi \sigma_{2}} \sigma_{1} e^{-i \varphi \sigma_{2}}=\cos 2 \varphi \sigma_{1}+\sin 2 \varphi \sigma_{3}, \\
e^{i \varphi \sigma_{2}} \sigma_{2} e^{-i \varphi \sigma_{2}}=\sigma_{2}, \\
e^{i \varphi \sigma_{2}} \sigma_{3} e^{-i \varphi \sigma_{2}}=-\sin 2 \varphi \sigma_{1}+\cos 2 \varphi \sigma_{3} ;
\end{gathered}
$$

that is,

$$
\mathscr{R}(\varphi)=\left[\begin{array}{ccc}
\cos 2 \varphi & 0 & -\sin 2 \varphi \\
0 & 1 & 0 \\
\sin 2 \varphi & 0 & \cos 2 \varphi
\end{array}\right] .
$$

The second entry of $\overline{\mathscr{H}}$ is unaffected and is rarely required in practical applications to nonadiabatic photochemistry, as the electronic states are often chosen real-valued. If so, the Hamiltonian matrix, $\mathbf{H}$, is a real symmetric matrix that depends only on three real parameters: $\mathscr{H}_{0}$ and the two-entry column-vector

$$
\widetilde{\mathscr{H}}=\left[\begin{array}{l}
H_{21}=H_{12} \\
\frac{H_{11}-H_{22}}{2}
\end{array}\right],
$$

which leads to the well-known expansion $[9,10]$ :

$$
\mathbf{H}=\frac{H_{11}+H_{22}}{2} \mathbf{1}+H_{12} \boldsymbol{\sigma}_{1}+\frac{H_{11}-H_{22}}{2} \boldsymbol{\sigma}_{3} .
$$

The corresponding restriction of $\mathscr{R}(\varphi)$ reads

$$
\widetilde{\mathscr{R}}(\varphi)=\left[\begin{array}{cc}
\cos 2 \varphi & -\sin 2 \varphi \\
\sin 2 \varphi & \cos 2 \varphi
\end{array}\right] .
$$

The similarity transform, $\mathbf{H}^{\prime}=\mathbf{U}^{\dagger}(\varphi) \mathbf{H U}(\varphi)$, is thus obtained from

$$
\mathscr{H}_{0}^{\prime}=\mathscr{H}_{0}, \quad \widetilde{\mathscr{H}}^{\prime}=\widetilde{\mathscr{R}}(\varphi) \widetilde{\mathscr{H}},
$$

which yields the well-known relationships used in nonadiabatic photochemistry for a two-state problem,

$$
\begin{gathered}
\frac{H_{11}^{\prime}+H_{22}^{\prime}}{2}=\frac{H_{11}+H_{22}}{2}, \\
H_{12}^{\prime}=\cos 2 \varphi H_{12}-\sin 2 \varphi \frac{H_{11}-H_{22}}{2}, \\
\frac{H_{11}^{\prime}-H_{22}^{\prime}}{2}=\sin 2 \varphi H_{12}+\cos 2 \varphi \frac{H_{11}-H_{22}}{2} .
\end{gathered}
$$

For real symmetric matrices $\mathbf{H}$ and rotations $\mathbf{U}(\varphi)$, the group homomorphism reduces to

$$
\begin{aligned}
& \mathrm{SO}(2) \longrightarrow \mathrm{SO}(2), \\
& \mathbf{U}^{\dagger}(\varphi) \longmapsto \mathscr{R}(\varphi),
\end{aligned}
$$


where $\mathscr{R}(\varphi) \equiv \mathbf{U}(2 \varphi)$. For obvious reasons, we also get $\mathbf{U}^{\dagger}(\varphi+\pi) \mapsto \mathscr{R}(\varphi)$, which is a manifestation of the doublevaluedness $[3,9,10,14-16]$ issue for such systems.

These relationships can be used, for example, when deriving the condition to be fulfilled by $\varphi$ to diagonalise $\mathbf{H}$,

$$
\mathbf{V}=\mathbf{U}^{\dagger}(\varphi) \mathbf{H U}(\varphi),
$$

where the adiabatic Hamiltonian matrix reads

$$
\mathbf{V}=\left[\begin{array}{cc}
V_{0} & 0 \\
0 & V_{1}
\end{array}\right]=\frac{V_{0}+V_{1}}{2} \mathbf{1}+\frac{V_{0}-V_{1}}{2} \boldsymbol{\sigma}_{3}
$$

with $V_{1}>V_{0}$ by convention. We define $\varphi$ such that the columns of $\mathbf{U}(\varphi)$ give the adiabatic states (eigenstates) in terms of the original states,

$$
\begin{gathered}
\left|\Psi_{0}\right\rangle=\cos \varphi\left|\Phi_{1}\right\rangle+\sin \varphi\left|\Phi_{2}\right\rangle, \\
\left|\Psi_{1}\right\rangle=-\sin \varphi\left|\Phi_{1}\right\rangle+\cos \varphi\left|\Phi_{2}\right\rangle,
\end{gathered}
$$

where the Schrödinger equation, for $\alpha=0,1$, reads

$$
\widehat{H}\left|\Psi_{\alpha}\right\rangle=V_{\alpha}\left|\Psi_{\alpha}\right\rangle \text {. }
$$

Hence,

$$
\begin{gathered}
\frac{V_{0}+V_{1}}{2}=\frac{H_{11}+H_{22}}{2}, \\
0=\cos 2 \varphi H_{12}-\sin 2 \varphi \frac{H_{11}-H_{22}}{2}, \\
\frac{V_{0}-V_{1}}{2}=\sin 2 \varphi H_{12}+\cos 2 \varphi \frac{H_{11}-H_{22}}{2},
\end{gathered}
$$

and rearranging the last two equations yields

$$
\begin{gathered}
\sin 2 \varphi=-\frac{H_{12}}{\sqrt{\left(\left(H_{11}-H_{22}\right) / 2\right)^{2}+\left(H_{12}\right)^{2}}}, \\
\cos 2 \varphi=-\frac{\left(H_{11}-H_{22}\right) / 2}{\sqrt{\left(\left(H_{11}-H_{22}\right) / 2\right)^{2}+\left(H_{12}\right)^{2}}}, \\
\frac{V_{0}-V_{1}}{2}=-\sqrt{\left(\left(H_{11}-H_{22}\right) / 2\right)^{2}+\left(H_{12}\right)^{2}}<0 .
\end{gathered}
$$

Alternatively, these can be used to generate an effective Hamiltonian matrix, $\mathbf{H}$, from the adiabatic Hamiltonian matrix $\mathbf{V}$ and a predefined angle. The inverse transformation, $\mathbf{U}^{\dagger}(\varphi)=\mathbf{U}(-\varphi)$, yields rotated states that span the same Hilbert subspace,

$$
\begin{aligned}
& \left|\Phi_{1}\right\rangle=\cos \varphi\left|\Psi_{0}\right\rangle-\sin \varphi\left|\Psi_{1}\right\rangle, \\
& \left|\Phi_{2}\right\rangle=\sin \varphi\left|\Psi_{0}\right\rangle+\cos \varphi\left|\Psi_{1}\right\rangle,
\end{aligned}
$$

and the corresponding effective Hamiltonian matrix reads

$$
\mathbf{H}=\mathbf{U}(\varphi) \mathbf{V U}^{\dagger}(\varphi)=\mathbf{U}^{\dagger}(-\varphi) \mathbf{V U}(-\varphi) .
$$

Hence,

$$
\begin{gathered}
\frac{H_{11}+H_{22}}{2}=\frac{V_{0}+V_{1}}{2}, \\
H_{12}=\sin 2 \varphi \frac{V_{0}-V_{1}}{2}, \\
\frac{H_{11}-H_{22}}{2}=\cos 2 \varphi \frac{V_{0}-V_{1}}{2} .
\end{gathered}
$$

This formulation is elegant and compact but is not required as such in order to derive the same relationships directly. However, it can become useful in situations where three states or more are coupled, as shown in the next section.

\section{Gell-Mann Matrices for Three-State Hamiltonian Matrices}

The Gell-Mann matrices $\left(\boldsymbol{\beta}_{m} \equiv \boldsymbol{\lambda}_{m}\right)$ are the analogue of Pauli matrices for $n=3$. They are traceless, Hermitian, and defined as

$$
\begin{array}{ll}
\lambda_{1}=\left[\begin{array}{lll}
0 & 1 & 0 \\
1 & 0 & 0 \\
0 & 0 & 0
\end{array}\right], & \lambda_{2}=\left[\begin{array}{ccc}
0 & -i & 0 \\
i & 0 & 0 \\
0 & 0 & 0
\end{array}\right], \\
\lambda_{3}=\left[\begin{array}{ccc}
1 & 0 & 0 \\
0 & -1 & 0 \\
0 & 0 & 0
\end{array}\right], & \lambda_{4}=\left[\begin{array}{lll}
0 & 0 & 1 \\
0 & 0 & 0 \\
1 & 0 & 0
\end{array}\right], \\
\lambda_{5}=\left[\begin{array}{ccc}
0 & 0 & -i \\
0 & 0 & 0 \\
i & 0 & 0
\end{array}\right], & \lambda_{6}=\left[\begin{array}{lll}
0 & 0 & 0 \\
0 & 0 & 1 \\
0 & 1 & 0
\end{array}\right], \\
\lambda_{7}=\left[\begin{array}{ccc}
0 & 0 & 0 \\
0 & 0 & -i \\
0 & i & 0
\end{array}\right], & \lambda_{8}=\frac{1}{\sqrt{3}}\left[\begin{array}{ccc}
1 & 0 & 0 \\
0 & 1 & 0 \\
0 & 0 & -2
\end{array}\right] .
\end{array}
$$

The corresponding $\mathbf{M} \in M_{3}(\mathbb{C}) \mapsto \mathscr{M} \in \mathbb{C}^{9}$ isomorphism is such that

$$
\begin{aligned}
& \mathbf{M} \\
& =\left[\begin{array}{lll}
M_{11} & M_{12} & M_{13} \\
M_{21} & M_{22} & M_{23} \\
M_{31} & M_{32} & M_{33}
\end{array}\right] \\
& =\left[\begin{array}{ccc}
\mathscr{M}_{0}+\mathscr{M}_{3}+\frac{1}{\sqrt{3}} \mathscr{M}_{8} & \mathscr{M}_{1}-i \mathscr{M}_{2} & \mathscr{M}_{4}-i \mathscr{M}_{5} \\
\mathscr{M}_{1}+i \mathscr{M}_{2} & \mathscr{M}_{0}-\mathscr{M}_{3}+\frac{1}{\sqrt{3}} \mathscr{M}_{8} & \mathscr{M}_{6}-i \mathscr{M}_{7} \\
\mathscr{M}_{4}+i \mathscr{M}_{5} & \mathscr{M}_{6}+i \mathscr{M}_{7} & \mathscr{M}_{0}-\frac{2}{\sqrt{3}} \mathscr{M}_{8}
\end{array}\right] \\
& \longleftrightarrow\left[\begin{array}{l}
\mathscr{M}_{0} \\
\mathscr{M}_{1} \\
\mathscr{M}_{2} \\
\mathscr{M}_{3} \\
\mathscr{M}_{4} \\
\mathscr{M}_{5} \\
\mathscr{M}_{6} \\
\mathscr{M}_{7} \\
\mathscr{M}_{8}
\end{array}\right]
\end{aligned}
$$




$$
\left[\begin{array}{c}
\frac{M_{11}+M_{22}+M_{33}}{3} \\
\frac{M_{12}+M_{21}}{2} \\
i \frac{M_{12}-M_{21}}{2} \\
\frac{M_{11}-M_{22}}{2} \\
\frac{M_{13}+M_{31}}{2} \\
i \frac{M_{13}-M_{31}}{2} \\
\frac{M_{23}+M_{32}}{2} \\
i \frac{M_{23}-M_{32}}{2} \\
\frac{M_{11}+M_{22}-2 M_{33}}{2 \sqrt{3}}
\end{array}\right]
$$

As required, for a complex Hermitian matrix $\mathbf{H}$, we get nine real parameters: $\mathscr{H}_{0}$ and

$$
\overline{\mathscr{H}}=\left[\begin{array}{c}
\mathfrak{R} H_{21}=\mathfrak{R} H_{12} \\
\mathfrak{J} H_{21}=-\mathfrak{J} H_{12} \\
\frac{H_{11}-H_{22}}{2} \\
\mathfrak{R} H_{31}=\mathfrak{R} H_{13} \\
\mathfrak{\Im} H_{31}=-\mathfrak{\Im} H_{13} \\
\mathfrak{R} H_{32}=\mathfrak{R} H_{23} \\
\mathfrak{\Im} H_{32}=-\mathfrak{\Im} H_{23} \\
\frac{H_{11}+H_{22}-2 H_{33}}{2 \sqrt{3}}
\end{array}\right] .
$$

The definition of the Gell-Mann matrices $\lambda_{3}$ and $\lambda_{8}$ seems to imply an arbitrary choice upon which the first two labels are not treated on the same footing as the third (by labels we mean the line and column indices of $\mathbf{M}$, e.g., the red, green, and blue colour charges of quarks in quantum chromodynamics). In fact, this apparent distinction hides a threefold equivalence where $\lambda_{3}$ and $\lambda_{8}$ form a degenerate irreducible representation of $E$-type in the threefold-rotation point group $C_{3}$ (e.g., $\lambda_{3}$ and $\lambda_{8}$ are the analogue of $e$-orbitals in $\mathrm{H}_{3}$ ). We thus propose to define conveniently four alternative linear combinations, which are not linearly independent from $\lambda_{3}$ and $\lambda_{8}$,

$$
\begin{aligned}
\lambda_{3}^{+} & =\left[\begin{array}{ccc}
0 & 0 & 0 \\
0 & 1 & 0 \\
0 & 0 & -1
\end{array}\right]=-\frac{1}{2} \lambda_{3}+\frac{\sqrt{3}}{2} \lambda_{8} \\
& =\cos \frac{2 \pi}{3} \lambda_{3}+\sin \frac{2 \pi}{3} \lambda_{8},
\end{aligned}
$$

$$
\begin{aligned}
\lambda_{8}^{+} & =\frac{1}{\sqrt{3}}\left[\begin{array}{ccc}
-2 & 0 & 0 \\
0 & 1 & 0 \\
0 & 0 & 1
\end{array}\right]=-\frac{\sqrt{3}}{2} \lambda_{3}-\frac{1}{2} \lambda_{8} \\
& =-\sin \frac{2 \pi}{3} \lambda_{3}+\cos \frac{2 \pi}{3} \lambda_{8}, \\
\lambda_{3}^{-} & =\left[\begin{array}{ccc}
-1 & 0 & 0 \\
0 & 0 & 0 \\
0 & 0 & 1
\end{array}\right]=-\frac{1}{2} \lambda_{3}-\frac{\sqrt{3}}{2} \lambda_{8} \\
& =\cos \frac{4 \pi}{3} \lambda_{3}+\sin \frac{4 \pi}{3} \lambda_{8}, \\
\lambda_{8}^{-} & =\frac{1}{\sqrt{3}}\left[\begin{array}{ccc}
1 & 0 & 0 \\
0 & -2 & 0 \\
0 & 0 & 1
\end{array}\right]=\frac{\sqrt{3}}{2} \lambda_{3}-\frac{1}{2} \lambda_{8} \\
& =-\sin \frac{4 \pi}{3} \lambda_{3}+\cos \frac{4 \pi}{3} \lambda_{8} .
\end{aligned}
$$

We now have three equivalent basis sets, $\overline{\mathscr{B}}=\left\{\lambda_{m}\right\}, \overline{\mathscr{B}}^{+}=$ $\left\{\boldsymbol{\lambda}_{m}^{+}\right\}$, and $\overline{\mathscr{B}}^{-}=\left\{\boldsymbol{\lambda}_{m}^{-}\right\}$(where $\boldsymbol{\lambda}_{m}^{ \pm}=\boldsymbol{\lambda}_{m}$ for $m \neq 3,8$ ). We further introduce

$$
\mathscr{T}=\left[\begin{array}{cccccccc}
1 & 0 & 0 & 0 & 0 & 0 & 0 & 0 \\
0 & 1 & 0 & 0 & 0 & 0 & 0 & 0 \\
0 & 0 & -\frac{1}{2} & 0 & 0 & 0 & 0 & \frac{\sqrt{3}}{2} \\
0 & 0 & 0 & 1 & 0 & 0 & 0 & 0 \\
0 & 0 & 0 & 0 & 1 & 0 & 0 & 0 \\
0 & 0 & 0 & 0 & 0 & 1 & 0 & 0 \\
0 & 0 & 0 & 0 & 0 & 0 & 1 & 0 \\
0 & 0 & -\frac{\sqrt{3}}{2} & 0 & 0 & 0 & 0 & -\frac{1}{2}
\end{array}\right],
$$

such that $\mathscr{T}$ and $\mathscr{T}^{2}=\mathscr{T}^{\mathrm{T}}=\mathscr{T}^{-1}$ are the corresponding threefold rotation matrices used to particularise labels 1 and 2 instead of 3 , respectively. Indeed,

$$
\begin{aligned}
& \overline{\mathscr{M}}_{+}=\mathscr{T} \overline{\mathscr{M}}, \\
& \overline{\mathscr{M}}_{-}=\mathscr{T}^{\mathrm{T}} \overline{\mathscr{M}},
\end{aligned}
$$

where $\mathscr{M}_{m}^{ \pm}=\mathscr{M}_{m}$ for $m \neq 3,8$ and

$$
\begin{gathered}
\mathscr{M}_{3}^{+}=\frac{M_{22}-M_{33}}{2}, \\
\mathscr{M}_{8}^{+}=\frac{M_{22}+M_{33}-2 M_{11}}{2 \sqrt{3}}, \\
\mathscr{M}_{3}^{-}=\frac{M_{33}-M_{11}}{2}, \\
\mathscr{M}_{8}^{-}=\frac{M_{33}+M_{11}-2 M_{22}}{2 \sqrt{3}} .
\end{gathered}
$$

We will later show how back and forth transformations among the three basis sets can be used as a trick that simplifies 
the treatment of similarity transformations for a three-state problem compared to the direct approach discussed by Yarkony and coworkers [5-7].

Gell-Mann matrices satisfy, for any $m_{1}, m_{2} \in\{1, \ldots, 8\}$,

$$
\boldsymbol{\lambda}_{m_{1}} \boldsymbol{\lambda}_{m_{2}}=\frac{2}{3} \delta_{m_{1} m_{2}} \mathbf{1}+\sum_{m_{3}=1}^{8}\left(d_{m_{1} m_{2} m_{3}}+i f_{m_{1} m_{2} m_{3}}\right) \boldsymbol{\lambda}_{m_{3}}
$$

where the nonzero structure constants are given by

$$
\begin{aligned}
& f_{\{123\}}=1 \text {, } \\
& f_{\{147\}}=-f_{\{156\}}=f_{\{246\}}=f_{\{257\}}=f_{\{345\}}=-f_{\{367\}}=\frac{1}{2}, \\
& f_{\{458\}}=f_{\{678\}}=\frac{\sqrt{3}}{2} .
\end{aligned}
$$

They are antisymmetric under the permutation of any pair of indices; for example, $f_{\{123\}}=f_{123}=-f_{213}=f_{231}=\cdots$. The nonzero elements of the $d$-coefficients are

$$
\begin{gathered}
d_{\{118\}}=d_{\{228\}}=d_{\{338\}}=-d_{\{888\}}=\frac{1}{\sqrt{3}}, \\
d_{\{448\}}=d_{\{558\}}=d_{\{668\}}=d_{\{778\}}=-\frac{1}{2 \sqrt{3}}, \\
d_{\{146\}}=d_{\{157\}}=-d_{\{247\}}=d_{\{256\}}=d_{\{344\}}=d_{\{355\}} \\
=-d_{\{366\}}=-d_{\{377\}}=\frac{1}{2} .
\end{gathered}
$$

In contrast, these are symmetric under the permutation of any pair of indices. As a consequence,

$$
\operatorname{tr}\left(\lambda_{m_{1}} \lambda_{m_{2}}\right)=2 \delta_{m_{1} m_{2}}
$$

and, for any $m_{1}, m_{2}, m_{3} \in\{1, \ldots, 8\}$,

$$
\operatorname{tr}\left(\boldsymbol{\lambda}_{m_{1}} \boldsymbol{\lambda}_{m_{2}} \boldsymbol{\lambda}_{m_{3}}\right)=2\left(d_{m_{1} m_{2} m_{3}}+i f_{m_{1} m_{2} m_{3}}\right)
$$

The corresponding multiplication table seems more complicated than in the case of SU(2). However, the threefold equivalence between the three basis sets corresponds to three underlying $\mathrm{SU}(2)$ subgroups embedded within $\mathrm{SU}(3)$, based on isomorphisms between Cartan subalgebras: $\left\{\sigma_{1}, \sigma_{2}, \sigma_{3}\right\} \leftrightarrow\left\{\lambda_{1}, \lambda_{2}, \lambda_{3}\right\} \leftrightarrow\left\{\lambda_{4},-\lambda_{5}, \lambda_{3}^{-}\right\} \leftrightarrow\left\{\lambda_{6}, \lambda_{7}, \lambda_{3}^{+}\right\}$. Note that $-\lambda_{5}$ occurs rather than $+\lambda_{5}$ in this mapping because this preserves "direct orientation" through circular permutations of the labels 1,2 , and 3 . We also introduce the $\mathrm{SU}(2)$-restricted identity matrices,

$$
\mathbf{e}=\left[\begin{array}{lll}
1 & 0 & 0 \\
0 & 1 & 0 \\
0 & 0 & 0
\end{array}\right]=\frac{2}{3} \mathbf{1}+\frac{1}{\sqrt{3}} \lambda_{8}
$$

TABLE 2: Multiplication table of Gell-Mann matrices $\left\{\boldsymbol{\lambda}_{1}, \boldsymbol{\lambda}_{2}, \boldsymbol{\lambda}_{3}, \boldsymbol{\lambda}_{8}\right\}$ (left times top).

\begin{tabular}{lccccc}
\hline Product & $\mathrm{e}$ & $\lambda_{1}$ & $\lambda_{2}$ & $\lambda_{3}$ & $\lambda_{8}$ \\
\hline $\mathrm{e}$ & $\mathrm{e}$ & $\lambda_{1}$ & $\lambda_{2}$ & $\lambda_{3}$ & $\frac{1}{\sqrt{3}} \mathrm{e}$ \\
$\lambda_{1}$ & $\lambda_{1}$ & $\mathrm{e}$ & $i \lambda_{3}$ & $-i \lambda_{2}$ & $\frac{1}{\sqrt{3}} \lambda_{1}$ \\
$\lambda_{2}$ & $\lambda_{2}$ & $-i \lambda_{3}$ & $\mathrm{e}$ & $i \lambda_{1}$ & $\frac{1}{\sqrt{3}} \lambda_{2}$ \\
$\lambda_{3}$ & $\lambda_{3}$ & $i \lambda_{2}$ & $-i \lambda_{1}$ & $\mathrm{e}$ & $\frac{1}{\sqrt{3}} \lambda_{3}$ \\
$\lambda_{8}$ & $\frac{1}{\sqrt{3}} \mathrm{e}$ & $\frac{1}{\sqrt{3}} \lambda_{1}$ & $\frac{1}{\sqrt{3}} \lambda_{2}$ & $\frac{1}{\sqrt{3}} \lambda_{3}$ & $\mathrm{e}-\frac{2}{\sqrt{3}} \lambda_{8}$ \\
\hline
\end{tabular}

TAble 3: Multiplication table of Gell-Mann matrices $\left\{\boldsymbol{\lambda}_{4}, \boldsymbol{\lambda}_{5}, \boldsymbol{\lambda}_{3}^{-}, \boldsymbol{\lambda}_{8}^{-}\right\}$ (left times top).

\begin{tabular}{lccccc}
\hline Product & $\mathrm{e}^{-}$ & $\lambda_{4}$ & $\lambda_{5}$ & $\lambda_{3}^{-}$ & $\lambda_{8}^{-}$ \\
\hline $\mathrm{e}^{-}$ & $\mathrm{e}^{-}$ & $\lambda_{4}$ & $\lambda_{5}$ & $\lambda_{3}^{-}$ & $\frac{1}{\sqrt{3}} \mathrm{e}^{-}$ \\
$\lambda_{4}$ & $\lambda_{4}$ & $\mathrm{e}^{-}$ & $-i \lambda_{3}^{-}$ & $i \lambda_{5}$ & $\frac{1}{\sqrt{3}} \lambda_{4}$ \\
$\lambda_{5}$ & $\lambda_{5}$ & $i \lambda_{3}^{-}$ & $\mathrm{e}^{-}$ & $-i \lambda_{4}$ & $\frac{1}{\sqrt{3}} \lambda_{5}$ \\
$\lambda_{3}^{-}$ & $\lambda_{3}^{-}$ & $-i \lambda_{5}$ & $i \lambda_{4}$ & $\mathrm{e}^{-}$ & $\frac{1}{\sqrt{3}} \lambda_{3}^{-}$ \\
$\lambda_{8}^{-}$ & $\frac{1}{\sqrt{3}} \mathrm{e}^{-}$ & $\frac{1}{\sqrt{3}} \lambda_{4}$ & $\frac{1}{\sqrt{3}} \lambda_{5}$ & $\frac{1}{\sqrt{3}} \lambda_{3}^{-}$ & $\mathrm{e}^{-}-\frac{2}{\sqrt{3}} \lambda_{8}^{-}$ \\
\hline
\end{tabular}

TABLE 4: Multiplication table of Gell-Mann matrices $\left\{\boldsymbol{\lambda}_{6}, \boldsymbol{\lambda}_{7}, \boldsymbol{\lambda}_{3}^{+}, \boldsymbol{\lambda}_{8}^{+}\right\}$ (left times top).

\begin{tabular}{lccccc}
\hline Product & $\mathrm{e}^{+}$ & $\lambda_{6}$ & $\lambda_{7}$ & $\lambda_{3}^{+}$ & $\lambda_{8}^{+}$ \\
\hline $\mathrm{e}^{+}$ & $\mathrm{e}^{+}$ & $\lambda_{6}$ & $\lambda_{7}$ & $\lambda_{3}^{+}$ & $\frac{1}{\sqrt{3}} \mathrm{e}^{+}$ \\
$\lambda_{6}$ & $\lambda_{6}$ & $\mathrm{e}^{+}$ & $i \lambda_{3}^{+}$ & $-i \lambda_{7}$ & $\frac{1}{\sqrt{3}} \lambda_{6}$ \\
$\lambda_{7}$ & $\lambda_{7}$ & $-i \lambda_{3}^{+}$ & $\mathrm{e}^{+}$ & $i \lambda_{6}$ & $\frac{1}{\sqrt{3}} \lambda_{7}$ \\
$\lambda_{3}^{+}$ & $\lambda_{3}^{+}$ & $i \lambda_{7}$ & $-i \lambda_{6}$ & $\mathrm{e}^{+}$ & $\frac{1}{\sqrt{3}} \lambda_{3}^{+}$ \\
$\lambda_{8}^{+}$ & $\frac{1}{\sqrt{3}} \mathrm{e}^{+}$ & $\frac{1}{\sqrt{3}} \lambda_{6}$ & $\frac{1}{\sqrt{3}} \lambda_{7}$ & $\frac{1}{\sqrt{3}} \lambda_{3}^{+}$ & $\mathrm{e}^{+}-\frac{2}{\sqrt{3}} \lambda_{8}^{+}$ \\
\hline
\end{tabular}

$$
\begin{aligned}
& \mathbf{e}^{-}=\left[\begin{array}{lll}
1 & 0 & 0 \\
0 & 0 & 0 \\
0 & 0 & 1
\end{array}\right]=\frac{2}{3} \mathbf{1}+\frac{1}{2} \lambda_{3}-\frac{1}{2 \sqrt{3}} \lambda_{8}=\frac{2}{3} \mathbf{1}+\frac{1}{\sqrt{3}} \lambda_{8}^{-}, \\
& \mathbf{e}^{+}=\left[\begin{array}{lll}
0 & 0 & 0 \\
0 & 1 & 0 \\
0 & 0 & 1
\end{array}\right]=\frac{2}{3} \mathbf{1}-\frac{1}{2} \lambda_{3}-\frac{1}{2 \sqrt{3}} \lambda_{8}=\frac{2}{3} \mathbf{1}+\frac{1}{\sqrt{3}} \lambda_{8}^{+} .
\end{aligned}
$$

For obvious reasons, $\lambda_{8}$ acts as $(1 / \sqrt{3}) \mathbf{e}$ with respect to $\left\{\lambda_{1}, \lambda_{2}, \lambda_{3}\right\}$ and thus commutes with them. Similar considerations apply to $\boldsymbol{\lambda}_{8}^{-},(1 / \sqrt{3}) \mathbf{e}^{-}$, and $\left\{\boldsymbol{\lambda}_{4},-\boldsymbol{\lambda}_{5}, \boldsymbol{\lambda}_{3}^{-}\right\}$, as well as $\lambda_{8}^{+},(1 / \sqrt{3}) \mathbf{e}^{+}$, and $\left\{\boldsymbol{\lambda}_{6}, \lambda_{7}, \lambda_{3}^{+}\right\}$. These relationships are summarised in Tables 2, 3, 4, 5, 6, and 7 . 
TABLE 5: Multiplication table of Gell-Mann matrices $\left\{\boldsymbol{\lambda}_{1}, \boldsymbol{\lambda}_{2}\right\}\left\{\boldsymbol{\lambda}_{4}, \boldsymbol{\lambda}_{5}\right.$, $\left.\lambda_{6}, \lambda_{7}\right\}$ (left times top).

\begin{tabular}{lcccc}
\hline Product & $\lambda_{4}$ & $\lambda_{5}$ & $\lambda_{6}$ & $\lambda_{7}$ \\
\hline$\lambda_{1}$ & $\frac{1}{2}\left(\lambda_{6}+i \lambda_{7}\right)$ & $-\frac{i}{2}\left(\lambda_{6}+i \lambda_{7}\right)$ & $\frac{1}{2}\left(\lambda_{4}+i \lambda_{5}\right)$ & $-\frac{i}{2}\left(\lambda_{4}+i \lambda_{5}\right)$ \\
$\lambda_{2}$ & $\frac{i}{2}\left(\lambda_{6}+i \lambda_{7}\right)$ & $\frac{1}{2}\left(\lambda_{6}+i \lambda_{7}\right)$ & $-\frac{i}{2}\left(\lambda_{4}+i \lambda_{5}\right)$ & $-\frac{1}{2}\left(\lambda_{4}+i \lambda_{5}\right)$ \\
\hline
\end{tabular}

TABLE 6: Multiplication table of Gell-Mann matrices $\left\{\boldsymbol{\lambda}_{4}, \boldsymbol{\lambda}_{5}\right\}\left\{\boldsymbol{\lambda}_{6}, \boldsymbol{\lambda}_{7}\right.$, $\left.\lambda_{1}, \lambda_{2}\right\}$ (left times top).

\begin{tabular}{lcccc}
\hline Product & $\lambda_{6}$ & $\lambda_{7}$ & $\lambda_{1}$ & $\lambda_{2}$ \\
\hline$\lambda_{4}$ & $\frac{1}{2}\left(\lambda_{1}+i \lambda_{2}\right)$ & $\frac{i}{2}\left(\lambda_{1}+i \lambda_{2}\right)$ & $\frac{1}{2}\left(\lambda_{6}-i \lambda_{7}\right)$ & $-\frac{i}{2}\left(\lambda_{6}-i \lambda_{7}\right)$ \\
$\lambda_{5}$ & $-\frac{i}{2}\left(\lambda_{1}+i \lambda_{2}\right)$ & $\frac{1}{2}\left(\lambda_{1}+i \lambda_{2}\right)$ & $\frac{i}{2}\left(\lambda_{6}-i \lambda_{7}\right)$ & $\frac{1}{2}\left(\lambda_{6}-i \lambda_{7}\right)$ \\
\hline
\end{tabular}

TABLE 7: Multiplication table of Gell-Mann matrices $\left\{\lambda_{6}, \lambda_{7}\right\}\left\{\lambda_{1}, \lambda_{2}\right.$, $\left.\lambda_{4}, \lambda_{5}\right\}$ (left times top).

\begin{tabular}{lcccc}
\hline Product & $\lambda_{1}$ & $\lambda_{2}$ & $\lambda_{4}$ & $\lambda_{5}$ \\
\hline$\lambda_{6}$ & $\frac{1}{2}\left(\lambda_{4}-i \lambda_{5}\right)$ & $\frac{i}{2}\left(\lambda_{4}-i \lambda_{5}\right)$ & $\frac{1}{2}\left(\lambda_{1}-i \lambda_{2}\right)$ & $\frac{i}{2}\left(\lambda_{1}-i \lambda_{2}\right)$ \\
$\lambda_{7}$ & $\frac{i}{2}\left(\lambda_{4}-i \lambda_{5}\right)$ & $-\frac{1}{2}\left(\lambda_{4}-i \lambda_{5}\right)$ & $-\frac{i}{2}\left(\lambda_{1}-i \lambda_{2}\right)$ & $\frac{1}{2}\left(\lambda_{1}-i \lambda_{2}\right)$ \\
\hline
\end{tabular}

We now consider the three elementary, direct rotations through the angles $(\varphi, \chi, \psi)$, represented with the three following orthogonal matrices:

$$
\begin{aligned}
& \mathbf{U}(\varphi)=\left[\begin{array}{ccc}
\cos \varphi & -\sin \varphi & 0 \\
\sin \varphi & \cos \varphi & 0 \\
0 & 0 & 1
\end{array}\right], \\
& \mathbf{U}(\chi)=\left[\begin{array}{ccc}
1 & 0 & 0 \\
0 & \cos \chi & -\sin \chi \\
0 & \sin \chi & \cos \chi
\end{array}\right], \\
& \mathbf{U}(\psi)=\left[\begin{array}{ccc}
\cos \psi & 0 & \sin \psi \\
0 & 1 & 0 \\
-\sin \psi & 0 & \cos \psi
\end{array}\right] .
\end{aligned}
$$

They can be expressed as

$$
\begin{aligned}
\mathbf{U}(\varphi) & =e^{-i \varphi \lambda_{2}}=\cos \varphi \mathbf{e}-i \sin \varphi \boldsymbol{\lambda}_{2}+\mathbf{1}-\mathbf{e} \\
& =\frac{2 \cos \varphi+1}{3} \mathbf{1}+\frac{\cos \varphi-1}{\sqrt{3}} \boldsymbol{\lambda}_{8}-i \sin \varphi \boldsymbol{\lambda}_{2}, \\
\mathbf{U}(\chi) & =e^{-i \chi \lambda_{7}}=\cos \chi \mathbf{e}^{+}-i \sin \chi \boldsymbol{\lambda}_{7}+\mathbf{1}-\mathbf{e}^{+} \\
& =\frac{2 \cos \chi+1}{3} \mathbf{1}+\frac{\cos \chi-1}{\sqrt{3}} \boldsymbol{\lambda}_{8}^{+}-i \sin \chi \boldsymbol{\lambda}_{7}, \\
\mathbf{U}(\psi) & =e^{i \psi \lambda_{5}}=\cos \psi \mathbf{e}^{-}+i \sin \psi \boldsymbol{\lambda}_{5}+\mathbf{1}-\mathbf{e}^{-} \\
& =\frac{2 \cos \psi+1}{3} \mathbf{1}+\frac{\cos \psi-1}{\sqrt{3}} \boldsymbol{\lambda}_{8}^{-}+i \sin \vartheta \boldsymbol{\lambda}_{5}
\end{aligned}
$$

and are such that $\mathbf{U}^{-1}(\vartheta)=\mathbf{U}^{\dagger}(\vartheta)=\mathbf{U}^{\mathrm{T}}(\vartheta)=\mathbf{U}(-\vartheta)$ for $\vartheta \in\{\varphi, \chi, \psi\}$. The corresponding similarity transformations for each basis matrix are given in Table 8 in the most adequate basis set. Only one is required, as the remaining two can be derived from it upon noticing the structural threefold equivalence between the three reordered basis sets, $\left\{\lambda_{1}, \lambda_{2}\right.$, $\left.\lambda_{3}, \lambda_{4},-\lambda_{5}, \lambda_{6}, \lambda_{7}, \lambda_{8}\right\} \leftrightarrow\left\{\lambda_{6}, \lambda_{7}, \lambda_{3}^{+}, \lambda_{1}, \lambda_{2}, \lambda_{4},-\lambda_{5}, \lambda_{8}^{+}\right\} \leftrightarrow$ $\left\{\lambda_{4},-\lambda_{5}, \lambda_{3}^{-}, \lambda_{6}, \lambda_{7}, \lambda_{1}, \lambda_{2}, \lambda_{8}^{-}\right\}$.

This yields three rotation matrices:

$\mathscr{R}(\varphi)$

$$
=\left[\begin{array}{cccccccc}
\cos 2 \varphi & 0 & -\sin 2 \varphi & 0 & 0 & 0 & 0 & 0 \\
0 & 1 & 0 & 0 & 0 & 0 & 0 & 0 \\
\sin 2 \varphi & 0 & \cos 2 \varphi & 0 & 0 & 0 & 0 & 0 \\
0 & 0 & 0 & \cos \varphi & 0 & \sin \varphi & 0 & 0 \\
0 & 0 & 0 & 0 & \cos \varphi & 0 & \sin \varphi & 0 \\
0 & 0 & 0 & -\sin \varphi & 0 & \cos \varphi & 0 & 0 \\
0 & 0 & 0 & 0 & -\sin \varphi & 0 & \cos \varphi & 0 \\
0 & 0 & 0 & 0 & 0 & 0 & 0 & 1
\end{array}\right],
$$

$\mathscr{R}_{+}(\chi)$

$$
=\left[\begin{array}{cccccccc}
\cos \chi & 0 & 0 & \sin \chi & 0 & 0 & 0 & 0 \\
0 & \cos \chi & 0 & 0 & \sin \chi & 0 & 0 & 0 \\
0 & 0 & \cos 2 \chi & 0 & 0 & \sin 2 \chi & 0 & 0 \\
-\sin \chi & 0 & 0 & \cos \chi & 0 & 0 & 0 & 0 \\
0 & -\sin \chi & 0 & 0 & \cos \chi & 0 & 0 & 0 \\
0 & 0 & -\sin 2 \chi & 0 & 0 & \cos 2 \chi & 0 & 0 \\
0 & 0 & 0 & 0 & 0 & 0 & 1 & 0 \\
0 & 0 & 0 & 0 & 0 & 0 & 0 & 1
\end{array}\right]
$$

$\mathscr{R}_{-}(\psi)$

$$
=\left[\begin{array}{cccccccc}
\cos \psi & 0 & 0 & 0 & 0 & -\sin \psi & 0 & 0 \\
0 & \cos \psi & 0 & 0 & 0 & 0 & \sin \psi & 0 \\
0 & 0 & \cos 2 \psi & \sin 2 \psi & 0 & 0 & 0 & 0 \\
0 & 0 & -\sin 2 \psi & \cos 2 \psi & 0 & 0 & 0 & 0 \\
0 & 0 & 0 & 0 & 1 & 0 & 0 & 0 \\
\sin \psi & 0 & 0 & 0 & 0 & \cos \psi & 0 & 0 \\
0 & -\sin \psi & 0 & 0 & 0 & 0 & \cos \psi & 0 \\
0 & 0 & 0 & 0 & 0 & 0 & 0 & 1
\end{array}\right] .
$$

The latter two can be transformed back into the original GellMann basis set, $\overline{\mathscr{B}}$, according to

$$
\begin{aligned}
& \mathscr{R}(\chi)=\mathscr{T}^{\mathrm{T}} \mathscr{R}_{+}(\chi) \mathscr{T}, \\
& \mathscr{R}(\psi)=\mathscr{T} \mathscr{R}_{-}(\psi) \mathscr{T}^{\mathrm{T}},
\end{aligned}
$$

which will prove to be a useful trick from an operational point of view.

All involved matrices (including $\mathscr{T}$ ) are rotation matrices, so that any composition of the three basic rotations of $\mathrm{SO}(3)$ yields a rotation matrix, the image of which in $\mathrm{SO}(8)$ is a rotation matrix. In other words, the aforementioned group homomorphism $\mathrm{U}^{\dagger} \in \mathrm{U}(3) \mapsto \mathscr{R} \in \mathrm{O}(8)$ can thus be 
TABLE 8: Similarity transforms of Gell-Mann matrices through elementary rotations.

\begin{tabular}{lccc}
\hline$\overline{\mathbf{H}}$ & $e^{i \varphi \lambda_{2}} \overline{\mathbf{H}} e^{-i \varphi \lambda_{2}}$ & $e^{i \chi \lambda_{7}} \overline{\mathbf{H}} e^{-i \chi \lambda_{7}}$ & $e^{-i \psi \lambda_{5}} \overline{\mathbf{H}} e^{i \psi \lambda_{5}}$ \\
\hline$\lambda_{1}$ & $\cos 2 \varphi \lambda_{1}+\sin 2 \varphi \lambda_{3}$ & $\cos \chi \lambda_{1}-\sin \chi \lambda_{4}$ & $-\sin \psi \lambda_{6}+\cos \psi \lambda_{1}$ \\
$\lambda_{2}$ & $\lambda_{2} \cos \psi \lambda_{2}$ \\
$\lambda_{4}$ & $\cos \varphi \lambda_{4}-\sin \varphi \lambda_{6}$ & $\cos \chi \lambda_{2}-\sin \chi \lambda_{5}$ & $\cos 2 \psi \lambda_{4}+\sin 2 \psi \lambda_{3}^{-}$ \\
$\lambda_{5}$ & $\cos \varphi \lambda_{5}-\sin \varphi \lambda_{7}$ & $\sin \chi \lambda_{1}+\cos \chi \lambda_{4}$ & $\lambda_{5}$ \\
$\lambda_{6}$ & $\sin \varphi \lambda_{4}+\cos \varphi \lambda_{6}$ & $\sin \chi \lambda_{2}+\cos \chi \lambda_{5}$ & $\cos \psi \lambda_{6}-\sin \psi \lambda_{1}$ \\
$\lambda_{7}$ & $\sin \varphi \lambda_{5}+\cos \varphi \lambda_{7}$ & $\cos 2 \chi \lambda_{6}+\sin 2 \chi \lambda_{3}^{+}$ & $\cos \psi \lambda_{7}+\sin \psi \lambda_{2}$ \\
$\lambda_{3}$ & $-\sin 2 \varphi \lambda_{1}+\cos 2 \varphi \lambda_{3}$ & $\lambda_{7}$ & $-\sin 2 \psi \lambda_{4}+\cos 2 \psi \lambda_{3}^{-}$ \\
$\lambda_{8}$ & $\lambda_{8}$ & & $\lambda_{8}^{-}$ \\
$\lambda_{3}^{-}$ & & $-\sin 2 \chi \lambda_{6}+\cos 2 \chi \lambda_{3}^{+}$ & $\lambda_{8}^{+}$ \\
$\lambda_{8}^{-}$ & & & \\
$\lambda_{3}^{+}$ & & & \\
$\lambda_{8}^{+}$ & & & \\
\hline
\end{tabular}

restricted to $\mathrm{SO}(3) \rightarrow \mathrm{SO}(8)$ (when $\mathrm{U}$ is chosen as a rotation matrix).

Let us now focus on the similarity transformation of a real symmetric matrix through a rotation. As already pointed out in the previous section, electronic states are often chosen realvalued in practice. Gell-Mann matrices can be partitioned into the set $\left\{\boldsymbol{\lambda}_{2}, \boldsymbol{\lambda}_{5}, \boldsymbol{\lambda}_{7}\right\}$, used to define rotations through matrix exponentiation (see above), and $\left\{\boldsymbol{\lambda}_{1}, \boldsymbol{\lambda}_{3}, \boldsymbol{\lambda}_{4}, \boldsymbol{\lambda}_{6}, \boldsymbol{\lambda}_{8}\right\}$, a basis set for real symmetric matrices. Any real symmetric matrix $\mathbf{H}$ is now given in terms of six real parameters: $\mathscr{H}_{0}$ and the five-entry column-vector (restricted to the basis set of real Gell-Mann matrices),

$$
\widetilde{\mathscr{H}}=\left[\begin{array}{c}
H_{21}=H_{12} \\
\frac{H_{11}-H_{22}}{2} \\
H_{31}=H_{13} \\
H_{32}=H_{23} \\
\frac{H_{11}+H_{22}-2 H_{33}}{2 \sqrt{3}}
\end{array}\right],
$$

$$
\begin{aligned}
\mathbf{U}(\psi, \theta, \phi) \\
=\mathbf{U}(\psi) \mathbf{U}(\theta) \mathbf{U}(\phi)=e^{i \psi \lambda_{2}} e^{i \theta \lambda_{7}} e^{i \phi \lambda_{2}} \\
=\left[\begin{array}{rrr}
\cos \psi \cos \phi-\sin \psi \cos \theta \sin \phi & \cos \psi \sin \phi+\sin \psi \cos \theta \cos \phi & \sin \psi \sin \theta \\
-\sin \psi \cos \phi-\cos \psi \cos \theta \sin \phi & -\sin \psi \sin \phi+\cos \psi \cos \theta \cos \phi & \cos \psi \sin \theta \\
\sin \theta \sin \phi & -\sin \theta \cos \phi & \cos \theta
\end{array}\right], \\
\mathbf{U}^{\dagger}(\psi, \theta, \phi)=\mathbf{U}^{\dagger}(\phi) \mathbf{U}^{\dagger}(\theta) \mathbf{U}^{\dagger}(\psi)=e^{-i \phi \lambda_{2}} e^{-i \theta \lambda_{7}} e^{-i \psi \lambda_{2}}=\mathbf{U}(-\phi,-\theta,-\psi) .
\end{aligned}
$$
$\mathbf{H}^{\prime}=\mathbf{U}^{\dagger} \mathbf{H U}$, is thus obtained from

$$
\mathscr{H}_{0}^{\prime}=\mathscr{H}_{0}, \quad \widetilde{\mathscr{H}}^{\prime}=\widetilde{\mathscr{R}} \widetilde{\mathscr{H}},
$$
on Cardan angles. three Euler angles $(\psi, \theta, \phi)$ as follows:

Note the isomorphism between $\left\{\boldsymbol{\lambda}_{2},-\boldsymbol{\lambda}_{5}, \boldsymbol{\lambda}_{7}\right\}$ and $\left\{\widehat{J}_{z}, \widehat{J}_{y}\right.$, $\left.\widehat{J}_{x}\right\}$ on the one hand and $\left\{\lambda_{1}, \lambda_{3}, \lambda_{4}, \lambda_{6},-\lambda_{8}\right\}$ and $\left\{d_{x y}, d_{x^{2}-y^{2}}\right.$, $\left.d_{x z}, d_{y z}, d_{z^{2}}\right\}$ on the other hand. The similarity transform,

where $\widetilde{\mathscr{R}}$ is the $5 \times 5$ restriction of $\mathscr{R}$ to real Gell-Mann matrices. At this stage, there are several possibilities for representing $\mathbf{U}$ as a given rotation of $\mathrm{SO}(3)$. We will consider two examples, one based on Euler angles, the other one based

As a first example, the rotation matrix is defined from 
The corresponding $5 \times 5$ matrix satisfies

$$
\begin{aligned}
\widetilde{\mathscr{R}}(\phi, \theta, \psi) & =\widetilde{\mathscr{R}}(\phi) \widetilde{\mathscr{R}}(\theta) \widetilde{\mathscr{R}}(\psi) \\
& =\widetilde{\mathscr{R}}(\phi) \widetilde{\mathscr{T}}^{\mathrm{T}} \widetilde{\mathscr{R}}_{+}(\theta) \widetilde{\mathscr{T}} \widetilde{\mathscr{R}}(\psi),
\end{aligned}
$$

where

$$
\begin{aligned}
& \widetilde{\mathscr{R}}(\psi)=\left[\begin{array}{ccccc}
\cos 2 \psi & \sin 2 \psi & 0 & 0 & 0 \\
-\sin 2 \psi & \cos 2 \psi & 0 & 0 & 0 \\
0 & 0 & \cos \psi & -\sin \psi & 0 \\
0 & 0 & \sin \psi & \cos \psi & 0 \\
0 & 0 & 0 & 0 & 1
\end{array}\right], \\
& \widetilde{\mathscr{R}}_{+}(\theta)=\left[\begin{array}{ccccc}
\cos \theta & 0 & -\sin \theta & 0 & 0 \\
0 & \cos 2 \theta & 0 & -\sin 2 \theta & 0 \\
\sin \theta & 0 & \cos \theta & 0 & 0 \\
0 & \sin 2 \theta & 0 & \cos 2 \theta & 0 \\
0 & 0 & 0 & 0 & 1
\end{array}\right],
\end{aligned}
$$

$$
\begin{aligned}
\mathbf{U}(\psi, \chi, \varphi) \\
=\mathbf{U}(\psi) \mathbf{U}(\chi) \mathbf{U}(\varphi)=e^{i \psi \lambda_{5}} e^{-i \chi \lambda_{7}} e^{-i \varphi \lambda_{2}} \\
=\left[\begin{array}{ccc}
\cos \psi \cos \varphi+\sin \psi \sin \chi \sin \varphi & -\cos \psi \sin \varphi+\sin \psi \sin \chi \cos \varphi & \sin \psi \cos \chi \\
\cos \chi \sin \varphi & \cos \chi \cos \varphi & -\sin \chi \\
-\sin \psi \cos \varphi+\cos \psi \sin \chi \sin \varphi & \sin \psi \sin \varphi+\cos \psi \sin \chi \cos \varphi & \cos \psi \cos \chi
\end{array}\right], \\
\mathbf{U}^{\dagger}(\psi, \chi, \varphi)=\mathbf{U}^{\dagger}(\varphi) \mathbf{U}^{\dagger}(\chi) \mathbf{U}^{\dagger}(\psi)=e^{i \varphi \lambda_{2}} e^{i \chi \lambda_{7}} e^{-i \psi \lambda_{5}} .
\end{aligned}
$$

The resulting entries of $\widetilde{\mathscr{R}}(\phi, \theta, \psi)$ are given in Table 9. They are in agreement with the entries of the matrix given in [5], except that the authors used different labels and scaling factors for their basis matrices,

$$
\begin{gathered}
\mathbf{B}^{(1)}=\lambda_{1}, \quad \mathbf{B}^{(2)}=\lambda_{4}, \quad \mathbf{B}^{(3)}=\lambda_{6}, \\
\mathbf{B}^{(4)}=\frac{1}{2} \lambda_{3}, \quad \mathbf{B}^{(5)}=-\frac{\sqrt{3}}{2} \lambda_{8} .
\end{gathered}
$$

Let us now consider Cardan angles $(\psi, \chi, \varphi)$ such that
The corresponding $5 \times 5$ matrix satisfies

$$
\begin{aligned}
\widetilde{\mathscr{R}}(\varphi, \chi, \psi) & =\widetilde{\mathscr{R}}(\varphi) \widetilde{\mathscr{R}}(\chi) \widetilde{\mathscr{R}}(\psi) \\
& =\widetilde{\mathscr{R}}(\varphi) \widetilde{\mathscr{T}}^{\mathrm{T}} \widetilde{\mathscr{R}}_{+}(\chi) \widetilde{\mathscr{T}} \widetilde{\mathscr{T}} \widetilde{\mathscr{R}}_{-}(\chi) \widetilde{\mathscr{T}}^{\mathrm{T}} \\
& =\widetilde{\mathscr{R}}(\varphi) \widetilde{\mathscr{T}}^{\mathrm{T}} \widetilde{\mathscr{R}}_{+}(\chi) \widetilde{\mathscr{T}}^{\mathrm{T}} \widetilde{\mathscr{R}}_{-}(\psi) \widetilde{\mathscr{T}}^{\mathrm{T}}
\end{aligned}
$$

where

$$
\widetilde{\mathscr{R}}_{-}(\psi)=\left[\begin{array}{ccccc}
\cos \psi & 0 & 0 & -\sin \psi & 0 \\
0 & \cos 2 \psi & \sin 2 \psi & 0 & 0 \\
0 & -\sin 2 \psi & \cos 2 \psi & 0 & 0 \\
\sin \psi & 0 & 0 & \cos \psi & 0 \\
0 & 0 & 0 & 0 & 1
\end{array}\right],
$$


Note that the last occurrence of $\widetilde{\mathscr{T}}^{\mathrm{T}}$ is irrelevant, as, in practice, one can consider a simpler transformation acting on $\widetilde{\mathscr{H}}_{-}=\widetilde{\mathscr{T}}^{\mathrm{T}} \widetilde{\mathscr{H}}$,

$$
\widetilde{\mathscr{R}}_{-}(\varphi, \chi, \psi)=\widetilde{\mathscr{R}}(\varphi) \widetilde{\mathscr{T}}^{\mathrm{T}} \widetilde{\mathscr{R}}_{+}(\chi) \widetilde{\mathscr{T}}^{\mathrm{T}} \widetilde{\mathscr{R}}_{-}(\psi),
$$

such that

$$
\widetilde{\mathscr{H}}^{\prime}=\widetilde{\mathscr{R}}_{-}(\varphi, \chi, \psi) \widetilde{\mathscr{H}}_{-},
$$

where

$$
\widetilde{\mathscr{H}}_{-}=\left[\begin{array}{c}
H_{12} \\
\frac{H_{33}-H_{11}}{2} \\
H_{13} \\
H_{23} \\
\frac{H_{33}+H_{11}-2 H_{22}}{2 \sqrt{3}}
\end{array}\right] .
$$

The resulting entries of $\widetilde{\mathscr{R}}_{-}(\varphi, \chi, \psi)$ are given in Table 10 .

These relationships can be used, for example, when deriving the conditions to be fulfilled by $(\psi, \chi, \varphi)$ to diagonalise $\mathbf{H}$,

$$
\mathbf{V}=\mathbf{U}^{\dagger}(\psi, \chi, \varphi) \mathbf{H U}(\psi, \chi, \varphi),
$$

where the adiabatic Hamiltonian matrix reads

$$
\begin{aligned}
\mathbf{V}=\left[\begin{array}{ccc}
V_{0} & 0 & 0 \\
0 & V_{1} & 0 \\
0 & 0 & V_{2}
\end{array}\right]= & \frac{V_{0}+V_{1}+V_{2}}{2} \mathbf{1}+\frac{V_{0}-V_{1}}{2} \boldsymbol{\lambda}_{3} \\
& +\frac{V_{0}+V_{1}-2 V_{2}}{2 \sqrt{3}} \boldsymbol{\lambda}_{8},
\end{aligned}
$$

with $V_{2}>V_{1}>V_{0}$ by convention. The Cardan angles are defined such that the columns of $\mathbf{U}(\psi, \chi, \varphi)$ give the adiabatic states (eigenstates) in terms of the original states. Hence, the three angles and the three eigenvalues satisfy six relationships,

$$
\begin{gathered}
\frac{V_{0}+V_{1}+V_{2}}{2}=\frac{H_{11}+H_{22}+H_{33}}{2} \\
{\left[\begin{array}{c}
0 \\
\frac{V_{0}-V_{1}}{2} \\
0 \\
0 \\
\frac{V_{0}+V_{1}-2 V_{2}}{2 \sqrt{3}}
\end{array}\right]=\widetilde{\mathscr{R}}_{-}(\varphi, \chi, \psi)\left[\begin{array}{c}
H_{12} \\
\frac{H_{33}-H_{11}}{2} \\
H_{13} \\
H_{23} \\
\frac{H_{33}+H_{11}-2 H_{22}}{2 \sqrt{3}}
\end{array}\right],}
\end{gathered}
$$

which can be expanded in terms of the entries of $\mathbf{H}$.

Reciprocally, this formulation can be used to generate an effective Hamiltonian matrix, $\mathbf{H}$, from the adiabatic Hamiltonian matrix $\mathbf{V}$ and predefined angles $(\psi, \chi, \varphi)$. The inverse transformation, $\mathbf{U}^{\dagger}(\psi, \chi, \varphi)$, yields rotated states that span the same Hilbert subspace. The corresponding effective Hamiltonian matrix reads

$$
\begin{aligned}
\mathbf{H} & =\mathbf{U}(\psi, \chi, \varphi) \mathbf{V} \mathbf{U}^{\dagger}(\psi, \chi, \varphi) \\
& =\left[\mathbf{U}^{\dagger}(\psi, \chi, \varphi)\right]^{\dagger} \mathbf{V} \mathbf{U}^{\dagger}(\psi, \chi, \varphi),
\end{aligned}
$$

which now involves $\widetilde{\mathscr{R}}_{-}^{\mathrm{T}}(\varphi, \chi, \psi)$, such that

$$
\begin{aligned}
& \frac{H_{11}+H_{22}+H_{33}}{2}=\frac{V_{0}+V_{1}+V_{2}}{2}, \\
& {\left[\begin{array}{c}
H_{12} \\
\frac{H_{33}-H_{11}}{2} \\
H_{13} \\
H_{23} \\
\frac{H_{33}+H_{11}-2 H_{22}}{2 \sqrt{3}}
\end{array}\right]} \\
& =\widetilde{\mathscr{R}}_{-}^{\mathrm{T}}(\varphi, \chi, \psi)\left[\begin{array}{c}
0 \\
\frac{V_{0}-V_{1}}{2} \\
0 \\
0 \\
\frac{V_{0}+V_{1}-2 V_{2}}{2 \sqrt{3}}
\end{array}\right] \\
& =\frac{V_{0}-V_{1}}{2} \\
& {\left[\begin{array}{c}
\sin 2 \varphi \cos \chi \cos \psi-\cos 2 \varphi \frac{\sin 2 \chi}{2} \sin \psi \\
-\sin 2 \varphi \sin \chi \sin 2 \psi+\cos 2 \varphi \frac{\cos 2 \chi-3}{4} \cos 2 \psi \\
\sin 2 \varphi \sin \chi \cos 2 \psi+\cos 2 \varphi \frac{\cos 2 \chi-3}{4} \sin 2 \psi \\
-\sin 2 \varphi \cos \chi \sin \psi-\cos 2 \varphi \frac{\sin 2 \chi}{2} \cos \psi \\
\cos 2 \varphi \frac{\sqrt{3}(\cos 2 \chi+1)}{4}
\end{array}\right]} \\
& +\frac{V_{0}+V_{1}-2 V_{2}}{2 \sqrt{3}} \\
& {\left[\begin{array}{c}
\frac{\sqrt{3} \sin 2 \chi}{2} \sin \psi \\
-\frac{\sqrt{3}(\cos 2 \chi+1)}{4} \cos 2 \psi \\
\frac{\sqrt{3}(\cos 2 \chi+1)}{4} \sin 2 \psi \\
-\frac{3 \sin 2 \chi}{2} \cos 2 \psi 1 \\
4
\end{array}\right] .}
\end{aligned}
$$


TABLE 9: Expressions of $\widetilde{\mathscr{R}}_{k, k^{\prime}}(\phi, \theta, \psi)$ for Euler angles $\left(k, k^{\prime} \in\{1,2,3,4,5\} \equiv m, m^{\prime} \in\{1,3,4,6,8\}\right)$.

\begin{tabular}{|c|c|c|c|c|}
\hline & $k=1$ & $\cos 2 \phi \cos \theta \cos 2 \psi-\sin 2 \phi \frac{3+\cos 2 \theta}{4} \sin 2 \psi$ & $k=2$ & $-\sin 2 \phi \cos \theta \cos 2 \psi-\cos 2 \phi \frac{3+\cos 2 \theta}{4} \sin 2 \psi$ \\
\hline$k^{\prime}=1$ & $\begin{array}{l}k=3 \\
k=5\end{array}$ & $\begin{array}{c}\cos \phi \sin \theta \cos 2 \psi-\sin \phi \frac{\sin 2 \theta}{2} \sin 2 \psi \\
-\frac{\sqrt{3}(1-\cos 2 \theta)}{4} \sin 2 \psi\end{array}$ & $k=4$ & $\sin \phi \sin \theta \cos 2 \psi+\cos \phi \frac{\sin 2 \theta}{2} \sin 2 \psi$ \\
\hline & $k=1$ & $\cos 2 \phi \cos \theta \sin 2 \psi+\sin 2 \phi \frac{3+\cos 2 \theta}{4} \cos 2 \psi$ & $k=2$ & $-\sin 2 \phi \cos \theta \sin 2 \psi+\cos 2 \phi \frac{3+\cos 2 \theta}{4} \cos 2 \psi$ \\
\hline$k^{\prime}=2$ & $\begin{array}{l}k=3 \\
k=5\end{array}$ & $\begin{array}{c}\cos \phi \sin \theta \sin 2 \psi+\sin \phi \frac{\sin 2 \theta}{2} \cos 2 \psi \\
\frac{\sqrt{3}(1-\cos 2 \theta)}{4} \cos 2 \psi\end{array}$ & $k=4$ & $\sin \phi \sin \theta \sin 2 \psi-\cos \phi \frac{\sin 2 \theta}{2} \cos 2 \psi$ \\
\hline$k^{\prime}=3$ & $\begin{array}{l}k=1 \\
k=3 \\
k=5\end{array}$ & $\begin{array}{c}-\cos 2 \phi \sin \theta \cos \psi+\sin 2 \phi \frac{\sin 2 \theta}{2} \sin \psi \\
\cos \phi \cos \theta \cos \psi-\sin \phi \cos 2 \theta \sin \psi \\
-\frac{\sqrt{3} \sin 2 \theta}{2} \sin \psi\end{array}$ & $\begin{array}{l}k=2 \\
k=4\end{array}$ & $\begin{array}{c}\sin 2 \phi \sin \theta \cos \psi+\cos 2 \phi \frac{\sin 2 \theta}{2} \sin \psi \\
\sin \phi \cos \theta \cos \psi+\cos \phi \cos 2 \theta \sin \psi\end{array}$ \\
\hline$k^{\prime}=4$ & $\begin{array}{l}k=1 \\
k=3 \\
k=5\end{array}$ & $\begin{array}{c}\cos 2 \phi \sin \theta \sin \psi+\sin 2 \phi \frac{\sin 2 \theta}{2} \cos \psi \\
-\cos \phi \cos \theta \sin \psi-\sin \phi \cos 2 \theta \cos \psi \\
-\frac{\sqrt{3} \sin 2 \theta}{2} \cos \psi\end{array}$ & $\begin{array}{l}k=2 \\
k=4\end{array}$ & $\begin{array}{l}-\sin 2 \phi \sin \theta \sin \psi+\cos 2 \phi \frac{\sin 2 \theta}{2} \cos \psi \\
-\sin \phi \cos \theta \sin \psi+\cos \phi \cos 2 \theta \cos \psi\end{array}$ \\
\hline$k^{\prime}=5$ & $\begin{array}{l}k=1 \\
k=3 \\
k=5\end{array}$ & $\begin{array}{c}\sin 2 \phi \frac{\sqrt{3}(1-\cos 2 \theta)}{4} \\
-\sin \phi \frac{\sqrt{3} \sin 2 \theta}{2} \\
\frac{1+3 \cos 2 \theta}{4}\end{array}$ & $\begin{array}{l}k=2 \\
k=4\end{array}$ & $\begin{array}{c}\cos 2 \phi \frac{\sqrt{3}(1-\cos 2 \theta)}{4} \\
\cos \phi \frac{\sqrt{3} \sin 2 \theta}{2}\end{array}$ \\
\hline
\end{tabular}

TABLE 10: Expressions of $\widetilde{\mathscr{R}}_{-k, k^{\prime}}(\varphi, \chi, \psi)$ for Cardan angles $\left(k, k^{\prime} \in\{1,2,3,4,5\} \equiv m, m^{\prime} \in\{1,3,4,6,8\}\right)$.

\begin{tabular}{|c|c|c|c|c|}
\hline & $k=1$ & $\cos 2 \varphi \cos \chi \cos \psi+\sin 2 \varphi \frac{\sin 2 \chi}{2} \sin \psi$ & $k=2$ & $\sin 2 \varphi \cos \chi \cos \psi-\cos 2 \varphi \frac{\sin 2 \chi}{2} \sin \psi$ \\
\hline$k^{\prime}=1$ & $\begin{array}{l}k=3 \\
k=5\end{array}$ & $\begin{array}{c}-\cos \varphi \sin \chi \cos \psi+\sin \varphi \cos 2 \chi \sin \psi \\
\frac{\sqrt{3} \sin 2 \chi}{2} \sin \psi\end{array}$ & $k=4$ & $\sin \varphi \sin \chi \cos \psi+\cos \varphi \cos 2 \chi \sin \psi$ \\
\hline$k^{\prime}=2$ & $\begin{array}{l}k=1 \\
k=3 \\
k=5\end{array}$ & $\begin{array}{c}-\cos 2 \varphi \sin \chi \sin 2 \psi-\sin 2 \varphi \frac{\cos 2 \chi-3}{4} \cos 2 \psi \\
-\cos \varphi \cos \chi \sin 2 \psi+\sin \varphi \frac{\sin 2 \chi}{2} \cos 2 \psi \\
-\frac{\sqrt{3}(\cos 2 \chi+1)}{4} \cos 2 \psi\end{array}$ & $\begin{array}{l}k=2 \\
k=4\end{array}$ & $\begin{array}{c}-\sin 2 \varphi \sin \chi \sin 2 \psi+\cos 2 \varphi \frac{\cos 2 \chi-3}{4} \cos 2 \psi \\
\sin \varphi \cos \chi \sin 2 \psi+\cos \varphi \frac{\sin 2 \chi}{2} \cos 2 \psi\end{array}$ \\
\hline$k^{\prime}=3$ & $\begin{array}{l}k=3 \\
k=5\end{array}$ & $\begin{array}{c}\cos 2 \varphi \sin \chi \cos 2 \psi-\sin 2 \varphi \frac{\cos 2 \chi-3}{4} \sin 2 \psi \\
\cos \varphi \cos \chi \cos 2 \psi+\sin \varphi \frac{\sin 2 \chi}{2} \sin 2 \psi \\
-\frac{\sqrt{3}(\cos 2 \chi+1)}{4} \sin 2 \psi\end{array}$ & $\begin{array}{l}k=2 \\
k=4\end{array}$ & $\begin{array}{c}\sin 2 \varphi \sin \chi \cos 2 \psi+\cos 2 \varphi \frac{\cos 2 \chi-3}{4} \sin 2 \psi \\
-\sin \varphi \cos \chi \cos 2 \psi+\cos \varphi \frac{\sin 2 \chi}{2} \sin 2 \psi\end{array}$ \\
\hline$k^{\prime}=4$ & $\begin{array}{l}k=1 \\
k=3 \\
k=5\end{array}$ & $\begin{array}{c}-\cos 2 \varphi \cos \chi \sin \psi+\sin 2 \varphi \frac{\sin 2 \chi}{2} \cos \psi \\
\cos \varphi \sin \chi \sin \psi+\sin \varphi \cos 2 \chi \cos \psi \\
\frac{\sqrt{3} \sin 2 \chi}{2} \cos \psi\end{array}$ & $\begin{array}{l}k=2 \\
k=4\end{array}$ & $\begin{array}{c}-\sin 2 \varphi \cos \chi \sin \psi-\cos 2 \varphi \frac{\sin 2 \chi}{2} \cos \psi \\
-\sin \varphi \sin \chi \sin \psi+\cos \varphi \cos 2 \chi \cos \psi\end{array}$ \\
\hline$k^{\prime}=5$ & $\begin{array}{l}k=1 \\
k=3 \\
k=5\end{array}$ & $\begin{array}{c}-\sin 2 \varphi \frac{\sqrt{3}(\cos 2 \chi+1)}{4} \\
\sin \varphi \frac{\sqrt{3} \sin 2 \chi}{2} \\
-\frac{3 \cos 2 \chi-1}{4}\end{array}$ & $\begin{array}{l}k=2 \\
k=4\end{array}$ & $\begin{array}{c}\cos 2 \varphi \frac{\sqrt{3}(\cos 2 \chi+1)}{4} \\
\cos \varphi \frac{\sqrt{3} \sin 2 \chi}{2}\end{array}$ \\
\hline
\end{tabular}




\section{Conclusions}

In this paper, we have recalled how a formulation based on Lie group homomorphisms can simplify the treatment of unitary similarity transformations of Hamiltonian matrices in nonadiabatic photochemistry from an operational perspective. For a two-state problem, this formulation based on Pauli matrices is elegant and compact but is not required as such. However, it can become useful in more complex situations, as first illustrated by Yarkony and coworkers for the symplectic group $\mathrm{Sp}(4) \subset \mathrm{SU}(4)$ connected to $\mathrm{SO}(5)$ in the case of a two-state conical intersection with an odd number of electrons [4] or for $\mathrm{O}(3)$ connected to $\mathrm{GL}(5, \mathbb{R})$ in the case of a three-state problem with no spin-orbit coupling [5-7]. In the latter case, we have reinvestigated their approach and shown that it can be rationalised in terms of Gell-Mann matrices. We confirmed their results for Euler angles and proposed another parameterisation based on Cardan angles. This formulation is general and can be extended to any $n$-state problem upon using $n^{2}-1$ generalised Pauli/Gell-Mann matrices for $n>3$ and a group homomorphism from $\mathrm{SU}(n)$ to $\mathrm{O}\left(n^{2}-1\right)$.

\section{Conflict of Interests}

The author declares that there is no conflict of interests regarding the publication of this paper.

\section{Acknowledgments}

The author is grateful to Fabien Gatti, David Lauvergnat, Aurelie Perveaux, and Michael A. Robb for their interest in this work.

\section{References}

[1] T. Pacher, L. S. Cederbaum, and H. Köppel, "Approximately diabatic states from block diagonalization of the electronic Hamiltonian," The Journal of Chemical Physics, vol. 89, no. 12, pp. 7367-7381, 1988.

[2] L. S. Cederbaum, J. Schirmer, and H.-D. Meyer, "Block diagonalisation of Hermitian matrices," Journal of Physics A: Mathematical and General, vol. 22, no. 13, pp. 2427-2439, 1989.

[3] C. A. Mead, "The geometric phase in molecular systems," Reviews of Modern Physics, vol. 64, no. 1, pp. 51-85, 1992.

[4] S. Han and D. R. Yarkony, "On the properties of the seam and branching spaces of conical intersections in molecules with an odd number of electrons: a group homomorphism approach," The Journal of Chemical Physics, vol. 118, no. 22, pp. 9952-9962, 2003.

[5] M. S. Schuurman and D. R. Yarkony, "On the characterization of three state conical intersections: a quasianalytic theory using a group homomorphism approach," The Journal of Chemical Physics, vol. 124, Article ID 124109, 2006.

[6] M. S. Schuurman and D. R. Yarkony, "On the characterization of three-state conical intersections using a group homomorphism approach: the two-state degeneracy spaces," The Journal of Physical Chemistry B, vol. 110, no. 38, pp. 19031-19039, 2006.

[7] M. S. Schuurman and D. R. Yarkony, "On the characterization of three state conical intersections using a group homomorphism approach: mapping the full $N-5$ dimensional seam space," The Journal of Chemical Physics, vol. 124, no. 24, Article ID 244103, 2006.

[8] W. Pauli Jr., "Zur quantenmechanik des magnetischen elektrons," Zeitschrift für Physik, vol. 43, no. 9-10, pp. 601-623, 1927.

[9] W. Domcke, D. R. Yarkony, and H. Köppel, Eds., Conical Intersections: Electronic Structure, Dynamics \& Spectroscopy, World Scientific, Singapore, 2004.

[10] W. Domcke, D. R. Yarkony, and H. Köppel, Eds., Conical Intersections: Theory, Computation and Experiment, World Scientific, Singapore, 2011.

[11] H. Weyl, The Classical Groups: Their Invariants and Representations, Princeton University Press, Princeton, NJ, USA, 1997.

[12] M. Gell-Mann and Y. Ne'eman, Eds., The Eightfold Way, W. A. Benjamin, New York, NY, USA, 1964.

[13] B. Hall, Lie Groups, Lie Algebras, and Representations-An Elementary Introduction, Springer, New York, NY, USA, 2003.

[14] M. V. Berry, "Quantal phase factors accompanying adiabatic changes," Proceedings of the Royal Society A, vol. 392, no. 1802, pp. 45-57, 1984.

[15] M. Baer, Beyond Born-Oppenheimer: Electronic Nonadiabatic Coupling Terms and Conical Intersections, Wiley, Hoboken, NJ, USA, 2006.

[16] S. Vanni, M. Garavelli, and M. A. Robb, "A new formulation of the phase change approach in the theory of conical intersections," Chemical Physics, vol. 347, no. 1-3, pp. 46-56, 2008. 


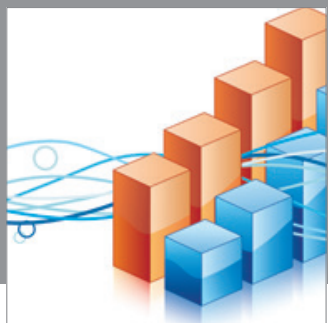

Advances in

Operations Research

mansans

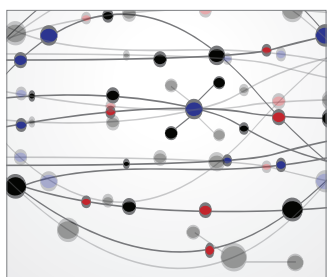

The Scientific World Journal
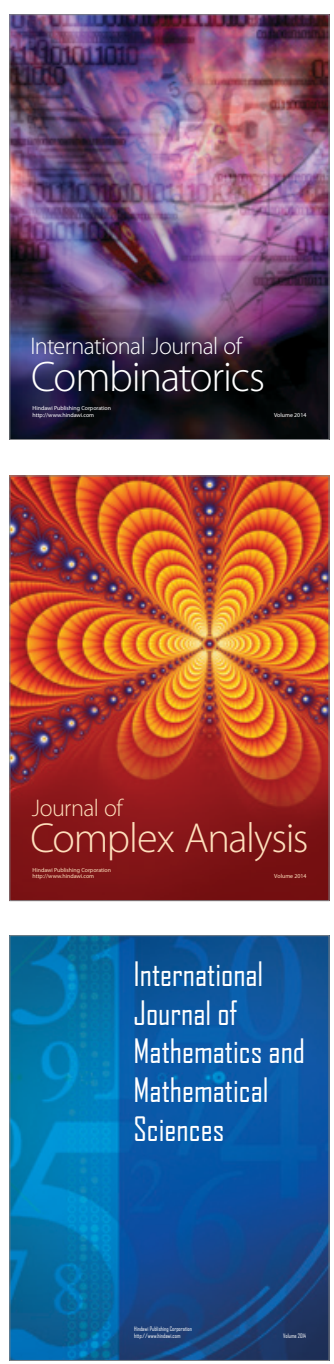
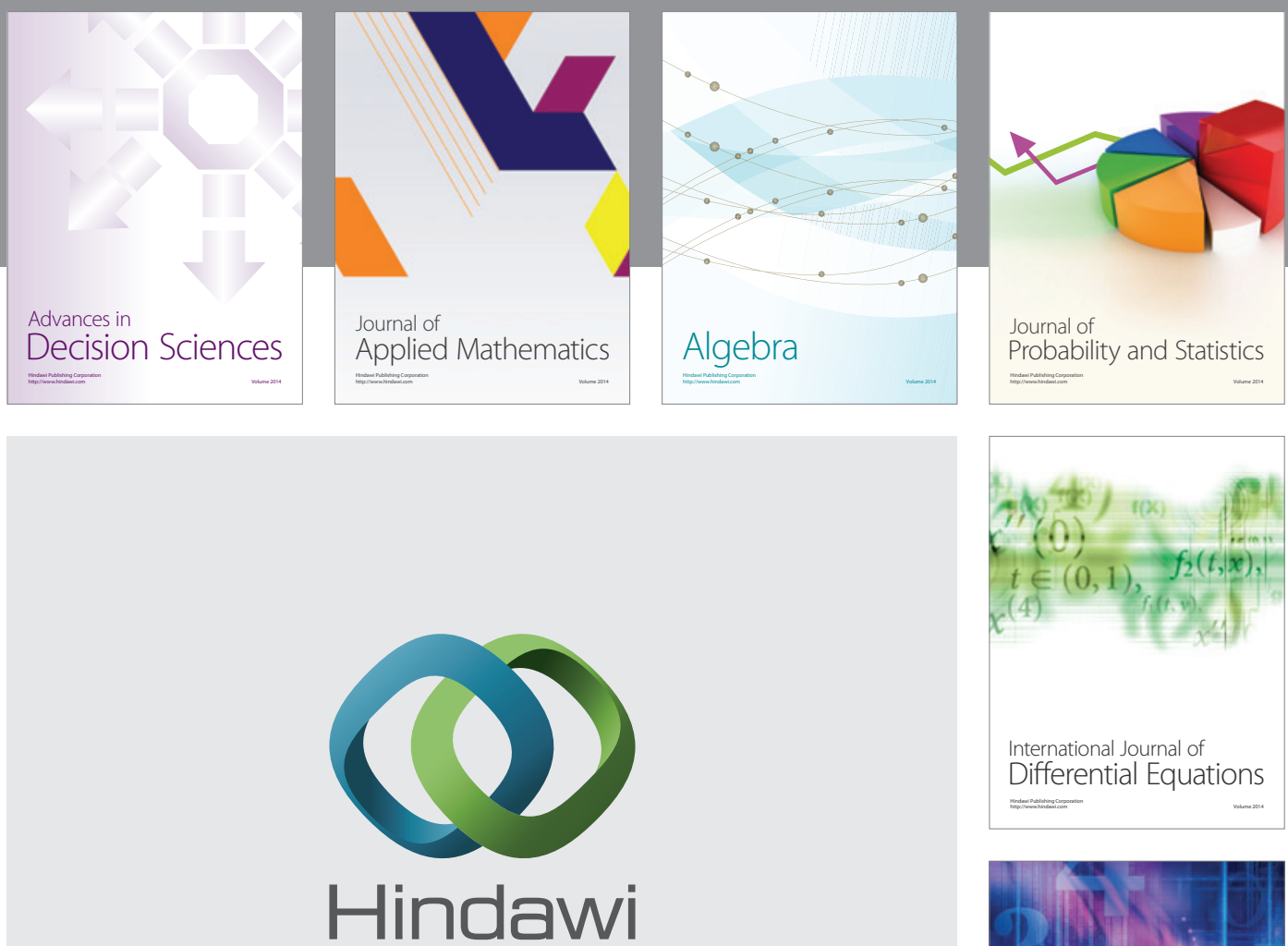

Submit your manuscripts at http://www.hindawi.com
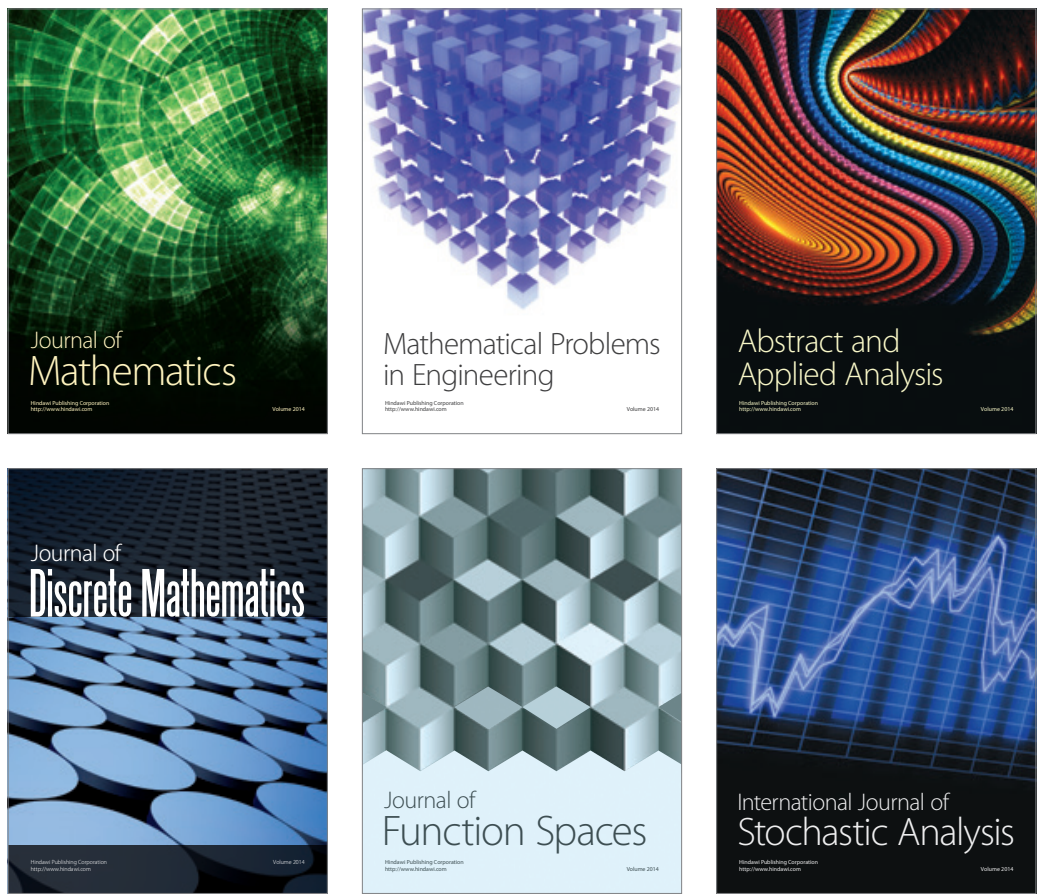

Journal of

Function Spaces

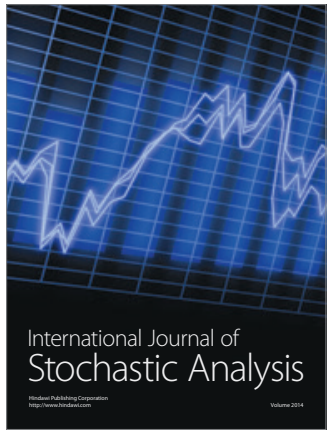

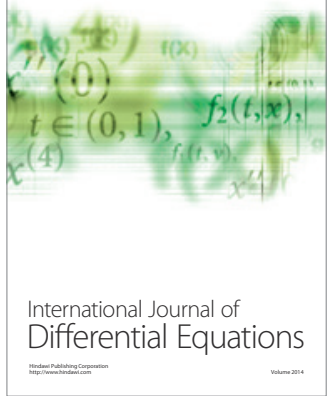
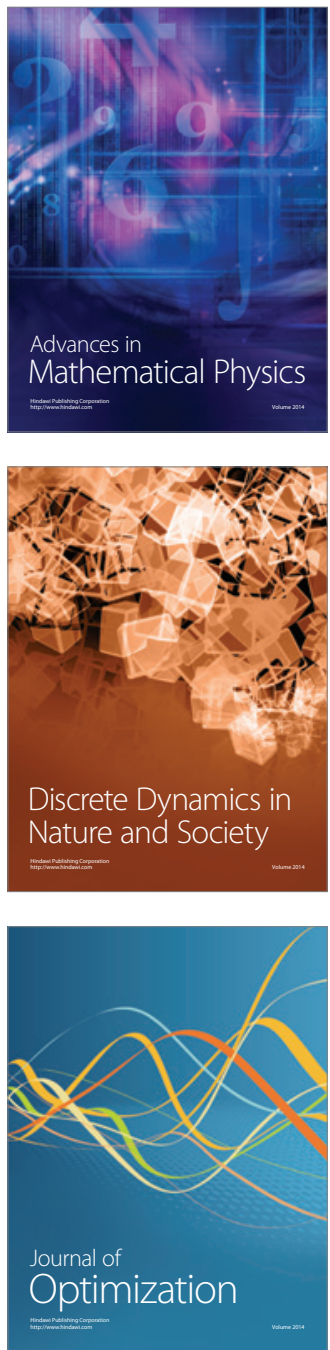